\title{
X-ray flares from Sgr A*: Star-disk interactions?
}

\author{
S. Nayakshin ${ }^{1}$, J. Cuadra $^{1}$, and R. Sunyaev ${ }^{1,2}$ \\ 1 Max-Planck-Institut für Astrophysik, Postfach 1317, 85741 Garching, Germany \\ 2 Space Research Institute, Moscow, Russia
}

Received 9 April 2003 / Accepted 16 September 2003

\begin{abstract}
Sgr A*, the putative black hole in our Galactic Center (GC), is extraordinary dim in all frequencies. Apparently the black hole is unable to accrete at the Bondi accretion rate for some reason. Another mystery of Sgr $\mathrm{A}^{*}$ is the recently discovered large magnitude and short duration X-ray flares (Baganoff et al. 2001). Here we point out that X-ray flares should be expected from star passages through an inactive (i.e. formerly accreting) disk. There are thousands of stars in Sgr $\mathrm{A}^{*}$ stellar cluster, and each star will pass through the disk twice per orbit. A shock hot enough to emit X-rays is formed around the star when the latter is inside the disk. We develop a simple yet somewhat detailed model for the X-ray emission from the shock. The duration of the flares, their X-ray spectra, frequency of events, and weakness of emission in the radio and near infra-red appear to be consistent with the available observations of X-ray flares. We therefore suggest that at least a fraction of the observed flares is due to the star-disk passages. Such star-disk flares are also of interest in the nuclei of nearby inactive galaxies, especially in connection with perspective Constellation-X and XEUS X-ray missions.
\end{abstract}

Key words. Galaxy: center - X-rays: galaxies - accretion, accretion disks - black hole physics

\section{Introduction}

\subsection{Sgr $A^{*}$ : Underfed X-ray flaring black hole}

With the current high quality data (e.g., Schödel et al. 2002; Genzel et al. 2003; Ghez et al. 2003a,b) it seems very safe to say that the center of our Galaxy hosts a supermassive black hole, with $M_{\mathrm{BH}} \simeq 3 \times 10^{6} M_{\odot}$. The black hole is associated with Sgr A* source, the compact radio core (Reid et al. 1999), discovered by Balick \& Brown (1974). Thanks to Chandra observations (Baganoff et al 2003a), we know that there is enough of hot gas at distances of the order $\sim 10^{17} \mathrm{~cm}$ (or $\sim 1^{\prime \prime}$ ) to make the black hole glow at around $\sim \mathrm{few} \times 10^{40} \mathrm{erg} \mathrm{s}^{-1}$ if accretion proceeds via the standard radiatively efficient disk (Shakura \& Sunyaev 1973). However the observed bolometric luminosity of $\mathrm{Sgr} \mathrm{A}^{*}$ is 4 orders of magnitude smaller than expected (e.g. Zhao et al. 2001; see also review by Melia \& Falcke 2001). The luminosity in X-rays is even smaller and is $\sim 10^{-7}$ of the nominally expected numbers (Baganoff et al. 2003a). Understanding of this discrepancy is the key to accretion physics at low accretion rates which encompasses a very large scope of objects from galactic centers to stellar mass compact objects (e.g., Narayan 2002).

The currently leading explanation of the low luminosity from Sgr A* is the Non-Radiative Accretion Flows (NRAFs), which were discussed in great detail first by Narayan \& Yi (1994). The corner stone assumption of this model - that ions

Send offprint requests to: S. Nayakshin, e-mail: serg@mpa-garching.mpg.de are much hotter than the electrons in the inner region of the flow - has not yet been independently verified. Initially it was believed that these flows carry ("advect") most of their energy into the black hole, radiating very little on the way. Several years ago it became clear that these hot flows also drive powerful thermal winds (Blandford \& Begelman 1999), so the accretion rate onto the black hole is significantly reduced. This theoretical picture seems to be confirmed in its gross features by the observations (e.g., Narayan et al. 1995; Bower et al. 2003; Quataert 2003), although radio jets may be an important additional ingredient of the model (Falcke \& Markoff 2000; Yuan et al. 2002). There are also alternative ideas that may explain the low luminosity of Sgr A* (e.g., Melia 1994; Coker \& Melia 2000; Proga \& Begelman 2003).

The recently discovered X-ray flares in Sgr A* (Baganoff et al. 2001) brought further theoretical challenges. The X-ray flares happen at a rate of about one per day (Goldwurm et al. 2003a; Baganoff et al. 2003b). The X-ray luminosity during the flares exceeds the quiescent one by up to a factor of 100 (although most of flares are weaker than that). Flare durations range from $\sim 1$ to $10 \mathrm{ks}$. The $\mathrm{X}$-ray spectrum of flares is harder than the quiescent one. The follow-up multi-wavelength campaign of Sgr $\mathrm{A}^{*}$ has not detected significant variability in any other band except for X-rays (Baganoff et al. 2003b; see also Hornstein et al. 2002). This latter fact is particularly troubling since in all the current models the X-ray flares occur at the inner part of the flow that emits Sgr $\mathrm{A}^{*}$ radio emission. Thus the challenge is to understand why such a large variation in X-rays does not lead to flares in other frequencies. 


\subsection{Cold disks in LLAGN and Sgr $A^{*}$}

It is believed that accretion flow physics at low accretion rates should be largely self-similar (e.g. Narayan 2002) and the difference is just in scales (e.g. $M_{\mathrm{BH}}$ and accretion rate $\dot{M}$ ). Therefore Sgr A* is expected to be physically similar to the Low Luminosity AGNs (LLAGN; e.g. Ho 1999). Most of LLAGN appear to posses thin cold disks. The presence of these disks is deduced convincingly through (i) water maser emission (Miyoshi et al. 1995); (ii) infrared "bumps" in the spectral energy distribution of LLAGN (Ho 1999) that are thought (Quataert et al. 1999) to be the thermal disk emission (with disk temperatures $T \lesssim 10^{3} \mathrm{~K}$ ); and (iii) the prevalence of the double-peaked broad emission lines (e.g. Ho 2003). The outer radii of these disks are poorly constrained, whereas the inner disk radii (from the SED and the emission line profiles) are in the range $\sim 100-1000 R_{\mathrm{S}}$, where $R_{\mathrm{S}}=2 G M_{\mathrm{BH}} / c^{2}$. For reference, $R_{\mathrm{S}} \simeq 9 \times 10^{11} \mathrm{~cm}$ is the Schwarzschild radius for the black hole mass of $M_{\mathrm{BH}}=3 \times 10^{6} M_{\odot}$.

Such a disk may also exist in our GC. There appear to be several ways to test the disk hypothesis: (i) through eclipses of close stars (Nayakshin \& Sunyaev 2003); and (ii) the reprocessing of the optical-UV radiation of these stars in the disk into the near infrared (NIR) band, which can yield a strong increase in the perceived NIR flux of the star. The current data alone rule out a disk optically thick in the NIR (Cuadra et al. 2003).

\subsection{X-ray flares in $\operatorname{Sgr} A^{*}$ : Star disk interactions?}

In this paper we point out an additional test of the inactive disk hypothesis. Recent results of Genzel et al. (2003) show that there are as many as $\sim 10^{4}$ stars in the inner arcsecond $\left(\sim 10^{5} R_{\mathrm{S}}\right)$ of Sgr A*. Several of these stars cross the inactive disk every day. While inside the disk, the stars drive strong and hot shock waves in the disk material, and emit X-rays. In this paper we shall build a simple yet somewhat detailed and selfcontained model for such X-ray flares.

Such star-disk flares have a potential to naturally explain why there are no counterparts of X-ray flares in the radio. The star-disk collisions typically occur at radii $R \gg 10 R_{\mathrm{S}}$, that is far from the region from which the radio emission originates. The problem of X-ray flares from star-disk passages merits a careful study even if there is no disk in our own Galaxy. Similar X-ray flares may be detectable in other LLAGN, especially the close ones such as M 31 or M 32 (Ho et al. 2003).

\section{Constraints on the disk properties}

\subsection{Arguments for and against a disk in Sgr $A^{*}$}

If the putative accretion disk were of the standard type (Shakura \& Sunyaev 1973), then it would have been seen by now even if accretion rate is as small as $10^{-9} M_{\odot} /$ year (Narayan 2002). However at very low disk accretion rates hydrogen in the disk becomes neutral, and the accretion rate plunges to very low values (e.g. see review by Cannizzo 1998; see also Eq. (12) below). This is why these disks are inactive, i.e. not accreting. In addition, these disks seem to miss their inner regions for a variety of reasons (e.g., Quataert et al. 1999 and Sect. 3.2) and therefore they can escape the near infra-red (NIR) detection limits that rule out standard steady-state disks.

Falcke \& Melia (1997; FM97 hereafter) probed the existence of the disk through its interaction with the winds from hot stars. This wind is believed to be the source of the hot gas that should be accreting on the black hole. FM97 assumed that the wind runs into and settles down onto the disk, releasing the mechanical energy as black-body radiation on impact. They found that unless the wind has a large specific angular momentum (so that it cannot penetrate to small radii), the luminosity expected in the NIR violates the available limits on Sgr A*, thus making the existence of the disk in Sgr $\mathrm{A}^{*}$ unlikely (see also Coker et al. 1999).

New data show that eleven out of twelve wind-producing stars rotate in the same (counter-Galaxy) direction (Genzel 2000). These and other hot luminous stars in the region share the same puzzling property: they are quite young (Ghez et al. 2003b; Genzel et al. 2003) and it is unclear how these stars made it to the innermost arcsecond of the Galaxy. One of the possibilities is that these stars were created from a single large molecular cloud that fell into the inner region of the Galaxy with a small impact parameter. This cloud could have created both the stars and an accretion disk whose (tiny not accreted) remnant may still be there ${ }^{1}$. Note also that matter in the accretion disk flows inward and outward from the characteristic (circularization) radius (Kolykhalov \& Sunyaev 1980). It is thus not possible to accrete all of the disk in the active phase without leaving a remnant.

Nayakshin (2003) recently showed that, in the setting similar to FM97, the hot flow rapidly looses its heat to the inactive disk. The latter re-radiates this energy in the infrared. As the hot gas looses heat, it also looses vertical pressure support, and as a result condenses onto the inactive disk. The accretion flow in the radial direction is essentially terminated. Thus the hot gas is accreting not on the black hole but rather on the inactive disk. Since the stellar wind sources are a few arcsecond (=few $\times 10^{5} R_{\mathrm{S}}$ ) away from Sgr A* (e.g. Genzel et al. 2003), the circularization radius is probably large (i.e. $\gtrsim 10^{4} R_{\mathrm{S}}$ ). The luminosity emitted by $\operatorname{Sgr} A^{*}$ is very small in this model because the gravitational potential at the condensation point is tiny compared with that of the last stable orbit around the black hole.

\subsection{Disk temperature (and NIR optical depth)}

First consider a disk optically thick in all frequencies. Let $R_{\mathrm{d}}$ be the outermost radius of the disk. Since the disk is inactive, the effective temperature of such a disk does not behave as the usual $T_{\mathrm{d}} \propto R^{-3 / 4}$. For example, $T_{\mathrm{d}}$ appears to stay nearly independent of radius for quiescent disks of eclipsing dwarf novae (e.g. Figs. 5 and 6 in Bobinger et al. 1997). If $T_{\mathrm{d}} \simeq$ const., then the disk total energy output is dominated by the emission from outermost radii. Accordingly the disk luminosity is

\footnotetext{
1 We have recently learned that, using data of R. Genzel's group, Levin \& Beloborodov (2003) found that 10 out of 13 wind-producing stars may lie "exactly" in a single plane, which further confirms our suggestions.
} 
$L_{\mathrm{d}} \sim 2 \pi R_{\mathrm{d}}^{2} \sigma_{B} T_{\mathrm{d}}^{4}$. In order to satisfy observational constraints on the total luminosity of $\mathrm{Sgr} \mathrm{A}^{*}$ in quiescence, $L_{\mathrm{q}}$, this temperature must be smaller than

$T_{\mathrm{d}}<\left[\frac{L_{\mathrm{q}}}{2 \pi R_{\mathrm{d}}^{2} \cos i \sigma_{B}}\right]^{1 / 4}=77 \mathrm{~K} \frac{L_{36}^{1 / 4}}{\cos i^{1 / 4} r_{4}^{1 / 2}}$,

where $L_{36}=L_{\mathrm{q}} / 10^{36} \mathrm{erg} \mathrm{s}^{-1}, r_{4}=R_{\mathrm{d}} / 10^{4} R_{\mathrm{S}}$. We shall see below that the disk is unlikely to be very optically thick so the disk midplane temperature should not exceed the effective temperature by a large number.

The disk should also re-radiate the visible and UV radiation of the $\mathrm{Sgr} \mathrm{A}^{*}$ star cluster itself. We use the latest results on the star number counts in Sgr $\mathrm{A}^{*}$ cluster from Genzel et al. (2003), together with the fact that the total luminosity of stars in the central parsec of the Galaxy is about 50 million Solar luminosities $\left(L_{\odot}\right)$ (Genzel 2000). We first calculate the light to mass ratio, and then use Eq. (15) to find that the integrated light of the star cluster within radius $R$ is

$L_{*}(R)=2.1 \times 10^{4} L_{\odot} r_{4}^{3 / 2} \mathrm{erg} \mathrm{s}^{-1}$.

The flux incident on the disk is then $F_{*}(R)=L_{*} / 4 \pi R^{2}$, and the resulting effective temperature due to stellar radiation heating is

$T_{*}=190 \mathrm{~K}_{4}^{-1 / 8}$,

which is a factor of 3 higher than the value in Eq. (1). This overproduces the infrared luminosity of Sgr $\mathrm{A}^{*}$ by about a factor of a hundred (e.g. see Sgr A* spectrum in Falcke \& Melia 2001; Narayan 2002). Therefore the disk must be optically thin in the infrared frequencies or highly inclined with respect to our line of sight. An estimate shows that for not too small disk inclination angles the infrared optical depth of the disk should be no larger than about 0.01 at frequency $\sim 10^{13} \mathrm{~Hz}$.

\subsection{Disk size}

The quiescent spectrum of Sgr $\mathrm{A}^{*}$ is in a rough accord with the data if the main part of the hot flow condenses onto the cold disk at radius $R_{\mathrm{c}} \gtrsim 3 \times 10^{4} R_{\mathrm{S}}$ (Nayakshin 2003). The value of $R_{\mathrm{c}}$ could be however reduced if the exact accretion rate in the condensing flow is lower than the Bondi estimate (due to arguments mentioned in Sect. 5.2 of Narayan 2002). Therefore we estimate the minimum disk outer radius as $R_{\mathrm{d}} \sim 1-10 \times$ $10^{4} R_{\mathrm{S}}$. There are no strong constraints based on the condensing flow model (Nayakshin 2003) on the inner disk radius, $R_{\mathrm{i}}$, as long as it is at least a factor of few smaller than $R_{\mathrm{c}}$.

\subsection{Constraints from close bright star passages}

Close bright stars could be eclipsed by the disk if it is optically thick in near infrared (Cuadra et al. 2003; Nayakshin \& Sunyaev 2003). Based on the measured S2 star's orbit (Schödel et al. 2002; Ghez et al. 2003b), if the disk is optically thick and has an inner hole less than $\sim 0.01^{\prime \prime} \sim 10^{3} R_{\mathrm{S}}$, then the outer disk radius may not much exceed $10^{4} R_{\mathrm{S}}$. However reprocessing of the optical-UV radiation received by the disk from the star into the NIR band could significantly increase the apparent star brightness. Using the deduced S2 orbit and its spectrum, Cuadra et al. (2003) finds that the disk, if any, must be optically thin in the NIR. An optically thin disk is allowed. Unfortunately these constraints are not easily converted into constraints on the total column depth of the disk, $\Sigma$ (see Sect. 2.8), because the properties of the dust grains in the disk are very uncertain (see Sect. 7). We also checked that the disk thermal emission, and that of the photo-ionized layer on the top of the disk, are negligible in both the radio and NIR frequencies.

\subsection{Minimum disk density}

Pressure equilibrium argument. In the frozen accretion model of Nayakshin (2003), the inactive disk and the hot accretion flow are in direct physical contact. Thus the disk midplane pressure should be at least as large as the pressure in the hot flow. Assuming the disk consists mainly of molecular hydrogen, $P_{\mathrm{d}} \simeq k n_{\mathrm{d}} T_{\mathrm{d}} / 2$, where $n_{\mathrm{d}}$ is the midplane density of hydrogen nuclei in $\mathrm{cm}^{-3}$. The density in the hot accretion flow can be estimated through $\dot{M} \sim 4 \pi R^{2} v_{R} m_{\mathrm{p}} n_{\text {hot }}$, where $v_{R} \sim \alpha \sqrt{G M_{\mathrm{BH}} / R}$ and $\alpha=0.1 \alpha_{1}$ is the viscosity parameter (Shakura \& Sunyaev 1973). This yields

$n_{\mathrm{d}} \gtrsim 1.5 \times 10^{9} \quad \mathrm{~cm}^{-3} \quad \frac{\dot{M}^{\prime} T^{\prime}}{\alpha_{1} T_{2}}\left(\frac{3 \times 10^{4} R_{\mathrm{S}}}{R_{\mathrm{c}}}\right)^{3 / 2}$,

where $\dot{M}^{\prime} \equiv \dot{M} / 3 \times 10^{-6} M_{\odot}$ year $^{-1}$ is the accretion rate in the hot flow, $T^{\prime}=T_{\text {hot }} / 3 \times 10^{7} \mathrm{~K}$ is dimensionless temperature of the hot flow, $R_{\mathrm{c}}$ is the wind circularization radius, and $T_{2}=$ $T_{\mathrm{d}} / 100 \mathrm{~K}$ is the disk unperturbed midplane temperature.

Total accreted mass argument. If condensation of the hot flow has been going on for a time $t_{\mathrm{a}}$, then the mass accreted by the disk from the wind is $\Delta M \sim \dot{M} t_{\mathrm{a}}$. If the accreted mass is deposited over area $\sim \pi R_{\mathrm{c}}^{2}$, then the accreted surface density is

$\Delta \Sigma_{\mathrm{d}} \sim 3 \mathrm{~g} / \mathrm{cm}^{2} \dot{M}^{\prime} t_{\mathrm{a} 6}\left(\frac{3 \times 10^{4} R_{\mathrm{S}}}{R_{\mathrm{c}}}\right)^{2}$,

where $t_{\mathrm{a} 6}=t_{\mathrm{a}} / 10^{6}$ years. This translates into the disk density increase of

$\Delta n_{\mathrm{d}} \sim 4 \times 10^{10} \mathrm{~cm}^{-3} \dot{M}^{\prime} t_{\mathrm{a} 6} T_{2}^{-1 / 2}\left(\frac{3 \times 10^{4} R_{\mathrm{S}}}{R_{\mathrm{c}}}\right)^{7 / 2}$.

This estimate yields a larger value for the minimum disk density than does Eq. (4), so we conclude that the minimum disk density is in the range of $\sim$ few $\times 10^{10}$ hydrogen nuclei in $\mathrm{cm}^{3}$. Note however that the above arguments are applicable to the disk radii with $R \sim R_{\mathrm{c}}$, and unfortunately they cannot be robustly extended to radii much smaller than that.

\subsection{Disk thickness}

The disk half thickness, $H$, is given by the hydrostatic balance (we neglect self-gravity; see below):

$H=\sqrt{\frac{k T R^{3}}{G M_{\mathrm{BH}} m_{\mathrm{p}}}}=3.9 \times 10^{12} T_{2}^{1 / 2} r_{4}^{3 / 2} \mathrm{~cm}$, 
where we assumed that the gas is dominated by molecular hydrogen so $\mu \simeq 2 m_{\mathrm{p}}$. Note that the star's radius $\left(\sim 10^{11} \mathrm{~cm}\right)$ is much smaller than the disk height scale for $R>10^{3} R_{\mathrm{S}}$. The disk is very flat since $H / R=0.96 \times 10^{-3} T_{2}^{1 / 2} r_{4}^{1 / 2}$.

\subsection{Mass of the disk and maximum density}

The mass of the accretion disk with a constant (with radius) midplane density and temperature, is

$M_{\mathrm{d}} \simeq 0.1 M_{\odot} n_{11}\left(\frac{R_{\mathrm{d}}}{10^{4} R_{\mathrm{S}}}\right)^{7 / 2} T_{2}^{1 / 2}$

where $n_{11} \equiv n_{\mathrm{d}} / 10^{11} \mathrm{~cm}^{-3}$ is the midplane density of hydrogen nuclei. $M_{\mathrm{d}}$ is much smaller than the black hole mass so that the disk is not globally self-gravitating (i.e., in the radial direction; Shlosman \& Begelman 1989) and will not gravitationally influence stellar dynamics in Sgr A*. The disk is locally (vertically) self-gravitating when $Q=M_{\mathrm{d}} /(H / R) M_{\mathrm{BH}} \gtrsim 1$ (Goldreich \& Lynden-Bell 1965; Kolykhalov \& Sunyaev 1980; Gammie 2001). We have

$Q=\frac{M_{\mathrm{d}}}{(H / R) M_{\mathrm{BH}}}=5.0 \times 10^{-5} r_{4}^{3} n_{11}$,

i.e. the disk is not vertically self-gravitating as well for the nominal values of $n_{\mathrm{d}}$ and $R_{\mathrm{d}}$ in this equation. We can turn the question around to derive the maximum disk density as the density at which the disk would become self-gravitating. In the latter case it would presumably break up into self-gravitating rings or regions and the star-disk impacts would become too rare. Hence,

$n_{\mathrm{d}} \lesssim 10^{15} r_{4}^{-3}$

For $r_{4}=3$ Eq. (10) yields maximum density $\sim$ few $\times 10^{13} \mathrm{~cm}^{-3}$. Note that Eq. (10) is applicable for any radius $r_{4}$ in contrast to Eqs. (4) and (6).

\subsection{Column depth and accretion rate}

The disk surface density is $\Sigma \simeq 2 H n_{\mathrm{d}} m_{\mathrm{p}}$, and is equal to

$\Sigma=1.3 n_{11} r_{4}^{3 / 2} T_{2}^{1 / 2} \mathrm{~g} \mathrm{~cm}^{-2}$

It is therefore clear that with the minimum and maximum disk density values just found, the disk can be either optically thin or optically thick to X-rays, and we should explore both cases.

We can also calculate the accretion rate through the disk as $\dot{M}_{\mathrm{d}} \simeq 2 \pi R \Sigma \alpha c_{\mathrm{s}}(H / R)^{2}$, which yields

$\dot{M}_{\mathrm{d}} \sim 10^{-10} \frac{M_{\odot}}{\text { year }} \alpha n_{11} r_{4}^{7 / 2} T_{2}^{2}$

Note that (i) this accretion rate is extremely small; (ii) it is not a constant with radius since the disk is not a steady state one and of course in a real disk density $n_{11}$ will vary with radius.

\subsection{Summary of disk properties}

Using quiescent $\operatorname{Sgr} \mathrm{A}^{*}$ spectral constraints, we found that the disk temperature should be $T_{\mathrm{d}} \sim 100 \mathrm{~K}$. The disk density should be small enough to preclude it from being selfgravitating and large enough to accommodate the mass deposition from the hot flow settling onto the disk. The respective maximum and minimum values of the disk density (according to Eqs. (6) and (10)) are $\sim$ few $\times 10^{13}$ and $\sim$ few $\times 10^{10} \mathrm{~cm}^{-3}$ at radius $R=3 \times 10^{4} R_{\mathrm{S}}$. If wind circularization radius is $R_{\mathrm{c}}=$ $10^{4} R_{\mathrm{S}}$ then these values increase to $\sim 10^{15}$ and $\sim 2 \times 10^{12} \mathrm{~cm}^{-3}$, respectively. With this range in disk density and also possibly large dynamical range between the inner and outer radii the disk can be either optically thin or thick in both X-rays and NIR. The disk has to be optically thin in the NIR frequencies (see Sect. 2.2).

\section{The role of the star cluster}

\subsection{The rate of star-disk crossings}

The distribution of stars in the central sub-parsec region of the Galaxy has been a subject of intense research for many years (see review by Genzel 2000). Existence of a stellar cusp with density distribution $\rho_{*}(R) \propto R^{-1.75}$ has been predicted by Bahcall \& Wolf (1976). Alexander (1999) found that a powerlaw stellar density distribution with exponent $p$ between 1.5 to 1.75 is statistically slightly favored over a constant density one. With unprecedentedly high statistic, Genzel et al. (2003) determined the stellar density distribution to follow the law

$\rho_{*}(R)=\left\{\begin{array}{l}\rho_{* 0}\left(R / R_{\mathrm{b}}\right)^{-p}, R<R_{\mathrm{b}} \\ \rho_{* 0}\left(R / R_{\mathrm{b}}\right)^{-2}, R_{\mathrm{b}}<R,\end{array}\right.$

where $R_{\mathrm{b}}=10 \operatorname{arcsec}, p=1.4 \pm 0.1$ and $\rho_{* 0}=1.2 \times$ $10^{6} M_{\odot} / \mathrm{pc}^{3}$. Note that $\rho_{* 0}=69 M_{\odot} / \operatorname{arcsec}^{3}$. This power-law distribution may persist down to the tidal disruption radius, $R_{\mathrm{t}}$, which is, according to Frank \& Rees (1976),

$R_{\mathrm{t}} \simeq 2.0 \times 10^{13} \mathrm{~cm} \mathrm{~m}_{*}^{-1 / 3} r_{*}$,

where $m_{*}$ is the mass of the star in Solar masses, and $r_{*}$ is stellar radius in units of Solar radius.

For convenience we choose $p=3 / 2$ as the resulting integrals are the simplest and the resulting accuracy is adequate for this paper. The mass of the stars within a given radius $R$ is

$M(R) \simeq 600 M_{\odot}\left(R_{\mathrm{b}} R\right)^{3 / 2}$,

where both $R_{\mathrm{b}}$ and $R$ are in units of arcseconds. For example, at $R=0.1,1$ and $10 \mathrm{arcsec}$, we have $\sim 600,2 \times 10^{4}$ and $6 \times$ $10^{5} M_{\odot}$, respectively.

Number of star-disk crossings per unit time and unit area is $n_{*}(R) \bar{v}_{\perp}(R) \simeq(1 / \sqrt{3}) n_{*}(R) v_{\mathrm{K}}(R)$ where $n_{*}(R)=\rho_{*}(R) / m_{*}$ and $\bar{v}_{\perp}(R)$ is the average of the star's velocity perpendicular to the disk, which we accepted to be $1 / \sqrt{3}$ of the local Keplerian value, $v_{\mathrm{K}}(R)$. Taking the integral over the disk surface area from the inner radius to the outer one, the number of star-disk crossings per unit time is

$\dot{N}(R)=(4 \pi / \sqrt{3}) \sqrt{G M_{\mathrm{BH}}} n_{* 0} R_{\mathrm{b}}^{3 / 2} \ln \frac{R_{\mathrm{d}}}{R_{\mathrm{i}}}=0.5 \ln \frac{R_{\mathrm{d}}}{R_{\mathrm{i}}} m_{*}^{-1}$ 
in units of day ${ }^{-1}$. If $R_{\mathrm{d}} / R_{\mathrm{i}}=500$, then this estimate yields $\sim 3$ star-disk crossings per day, whereas if $R_{\mathrm{d}} / R_{\mathrm{i}}=10$ then we have $\sim 1$ crossing per day. The estimate (16) is very close to the observed rate of the X-ray flares of about 1 flare per day (Baganoff et al. 2003b).

\subsection{Effects of the stellar cluster on the disk}

Under the assumption that the disk is truly inactive, i.e. its viscosity is zero, the only mechanism for a radial mass transfer in the disk is the angular momentum loss due to the star-disk collisions. Ostriker (1983) calculated the rate of the angular momentum loss by the disk assuming an isotropic star cluster. We will use his results for the Bahcall-Wolf (1976) distribution of stars since the latter yields $n_{*}(R) \propto R^{-7 / 4}$, i.e. roughly same as observed (Genzel et al. 2003). For relatively small radii that we are interested in, i.e., $R \lesssim 10^{4} R_{\mathrm{S}}$, the angular momentum transfer from the star to the disk is dominated by the hydrodynamical interaction (rather than by tidal effects) and so we set $\eta=0$ (see Eq. (10) in Ostriker 1983). Ostriker (1983) defined the "drag time" $t_{\mathrm{d}}$ as the time for the disk at radius $R$ to loose all of its angular momentum due to the star-disk collisions. For the Bahcall-Wolf (1976) distribution,

$t_{\mathrm{d}}=\frac{0.09}{n_{*}(R) R_{*}^{2} v_{k}(R)}$.

Note that $t_{\mathrm{d}} \propto R^{2}$, and therefore it is always the inner disk that can be destroyed most rapidly (accreted onto the black hole) due to the star-disk collisions.

As in Sect. 2.5, suppose that the age of the inactive disk is $t_{\mathrm{a}}=10^{6} t_{\mathrm{a} 6}$ years. Then the disk regions with $t_{\mathrm{d}}(R) \lessgtr t_{\mathrm{a}}$ will be significantly affected by the star-disk passages and thus we can define the inner inactive disk radius $R_{\mathrm{ii}}$ through $t_{\mathrm{d}}\left(R_{\mathrm{ii}}\right)=t_{\mathrm{a}}$ :

$R_{\mathrm{ii}}=R_{*} \sqrt{\frac{\left.\dot{N}_{(} R_{\mathrm{ii}}\right) t_{\mathrm{a}}}{0.092 \pi \ln R_{\mathrm{ii}} / R_{\mathrm{t}}}}=2.2 \times 10^{3} R_{\mathrm{S}} r_{*} \sqrt{\dot{N}_{3} t_{6}}$,

where $\dot{N}_{3}$ is the star-disk collision rate in units of $10^{3}$ year $^{-1}$, and where we set $R_{\mathrm{ii}}=10^{3} R_{\mathrm{S}}$ in the slowly changing logarithmic factor. Note that the factor in the denominator of Eq. (18) is $\sim 4$ so that a simple interpretation of the result is that the disk area within radius $R_{\mathrm{ii}}$ approximately equals to the area of the disk covered by the star-disk collisions in $t_{\mathrm{a}}$ years. It is also worth observing that $R_{\mathrm{ii}}$ depends relatively weakly on the stardisk collision rate $\dot{N}$ and $t_{\mathrm{a}}$.

Finally, the inner disk is also strongly affected by means other than direct stellar impacts. For example, the S2 star is as luminous as $10^{5}$ Solar luminosities (Ghez et al. 2003b), and it came to within $2 \times 10^{3} R_{\mathrm{S}}$ of the black hole (Schödel et al. 2002). Clearly its separation from the disk surface would be very small at the time of the closest approach and the disk is heated to temperatures as much as $10^{3} \mathrm{~K}$. The disk may become ionized enough to actually start accreting onto the black hole (at these small radii only).

Therefore we conclude that the putative disk in $\mathrm{Sgr} \mathrm{A}^{*}$ is likely to have two physically distinct zones. The inner zone with radius $R_{\mathrm{i}}<R<R_{\mathrm{ii}}$ is likely to be hotter than the inactive disk discussed in Sect. 2, with angular momentum transfer dominated by stellar impacts. The accretion rate through this disk would have to be quite small to comply with $\mathrm{Sgr} \mathrm{A}^{*}$ detection limits.

\subsection{Summary: The role of the Sgr $A^{*}$ cluster}

We have shown (Sect. 3.1) that the rate of star-disk crossings is few per day for the stellar cusp distribution given by Genzel et al. (2003) This calculated event rate is rather close to what is observed ( 1 flare per day). In addition, we found that the star impacts on the inner disk are so frequent for $R \lessgtr 10^{3} R_{\mathrm{S}}$ that the inner disk may be simply emptied out, i.e. accreted onto the black hole. The inner inactive disk radius was estimated to be $R_{\mathrm{ii}} \sim 10^{3} R_{\mathrm{S}}$. Below that radius the flow pattern will have to change to reflect the too frequent stellar passages. Most likely the disk will be hotter than it is at $R>R_{\mathrm{ii}}$.

\section{Schematics of a star passage through the disk}

\subsection{Background notes}

The subject of star-disk interactions in AGN has been extensively studied in the literature. There were two main themes in these studies. One was the influence of the stars on the disk evolution through the additional drag imposed by the stars on the disk (e.g. Ostriker 1983; Norman \& Silk 1983; Hagio 1987, see also Sect. 3.2), heating (e.g. Perry \& Williams 1993) and mass deposition due to stripping and ablation of star's envelopes (Armitage et al. 1996). Not less important were the studies of the influence of the disk on the distribution of the stellar orbits in the star cluster (e.g. Norman \& Silk 1983; Vilkoviskij 1983; Syer et al. 1991; Rauch 1995; Karas \& Subr 2001; Vilkoviskij \& Czerny 2002). Zurek et al. (1994) proposed that the wake region behind the star (when the star leaves the disk) may be the putative "broad line region clouds" of AGN. Ivanov et al. (1998) studied the passage of a black hole through the accretion disk. At the same time relatively little attention has been given to the emission from a single star-disk passage. The point is that this emission is usually weak on the background of the AGN and is unlikely to be seen, although the effects of many star-disk collisions may be observable (Zentsova 1983; Perry \& Williams 1993).

Furthermore, the optical depth of the dense AGN-type disks is enormous, and thus no X-rays would make it out of the disk. Thus X-ray emission from such star-disk passages was not expected. However, in the case of $\mathrm{Sgr} \mathrm{A}^{*}$ the disk density is probably much lower than the typical values considered in the literature on star-disk collisions so far. In addition, even if the disk midplane density is high enough so that the disk is optically thick, density becomes quite small in the disk photosphere. The $\mathrm{X}$-ray emission is too weak in the context of normal AGN, but not so for Sgr $\mathrm{A}^{*}$ due to its dimness and proximity.

\subsection{Phases of the star passage through the disk}

The star's internal density is very much larger than that of the disk and so effects of the disk on the star during one passage are minimal (see Syer et al. 1991). An alternative way to see this 

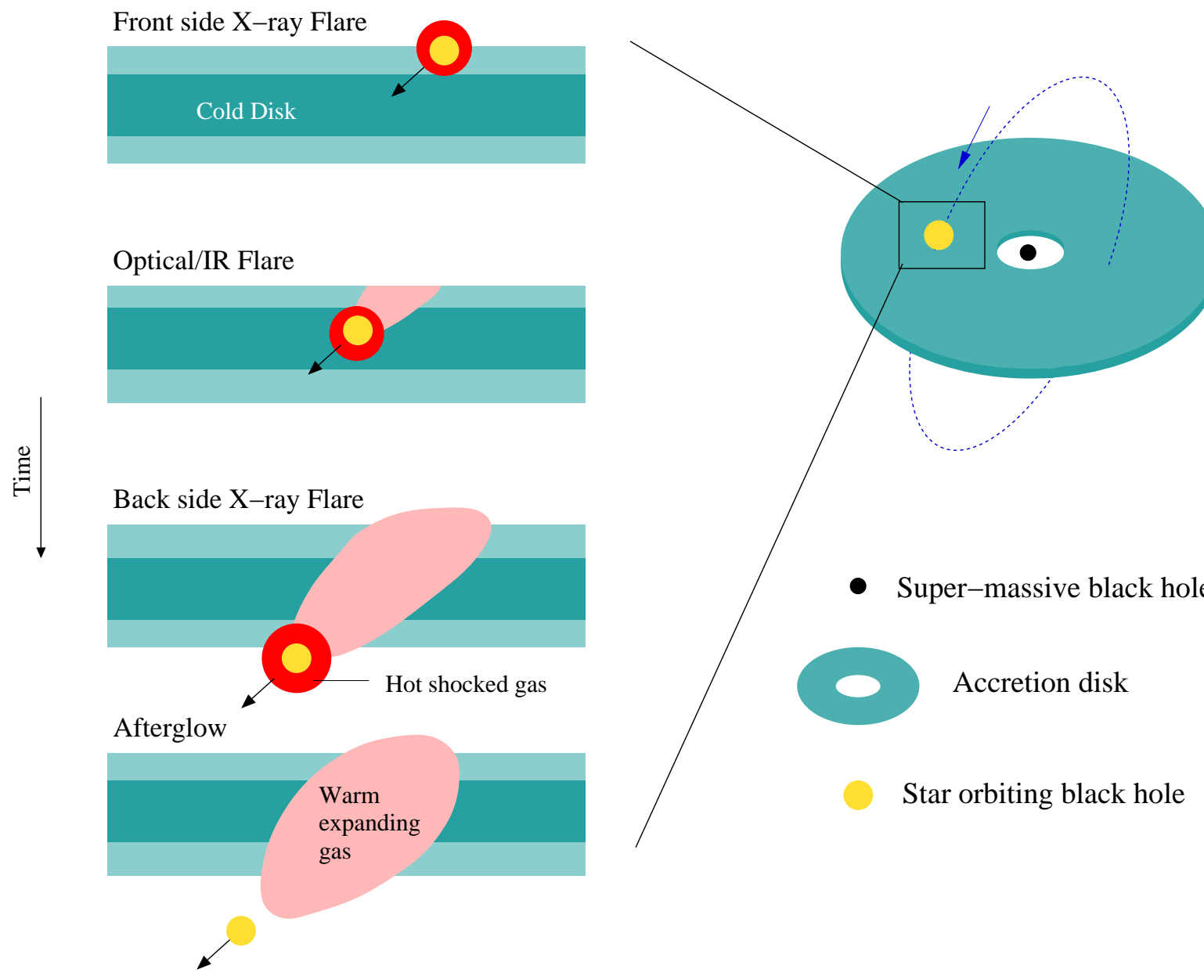

Fig. 1. Cartoon of a low mass star striking the accretion disk around the black hole and producing the flare. The inset on the left shows sequence of events when the star passes through the disk. The star is shown as a filled yellow circle with an arrow. The disk is schematically divided into the optically thick (darker) and the optically thin - the photosphere - parts (lighter color). Time runs top to bottom. The X-rays are observable only when the star is in the photosphere of the disk (first and third sketches from the top). When the star is deep inside the disk (second from the top sketch), the emitted X-rays are absorbed, thermalized and re-emitted in the optical or near infra-red. In the afterglow phase (bottom) the hot shocked gas cooled to low temperatures and only weak infra-red emission is present.

is to compare the mass of the cold gas swept up by the star as it travels through the disk, to the stellar mass $\left(M_{*}\right): \Delta M / M_{*}=$ $\Sigma \pi R_{*}^{2} / M_{*}=1.4 \times 10^{-11} n_{11} T_{2}^{1 / 2} r_{4}^{3 / 2} r_{*}^{2} m_{*}^{-1} \ll 1$. We thus consider the star to be a rigid solid body in this paper. We can also neglect accretion of gas onto the stellar surface because the corresponding Bondi radius is much smaller than $R_{*}: R_{\mathrm{B}}=$ $G M_{*} m_{\mathrm{p}} / 2 k T_{\text {char }}(R)=4.5 \times 10^{9} m_{*} r_{4} \ll 7 \times 10^{10} r_{*}$, where $T_{\text {char }}$ is the characteristic temperature defined by Eq. (23), and is close to the local virial temperature. Tidal interactions of the star and the gas in the wake of the star are hence neglected.

The star drives a shock into the disk. The Mach number is very large, $v_{*} / c_{\text {disk }} \sim \sqrt{T_{\text {char }} / T_{\mathrm{d}}} \sim 10^{3}$, and thus the star drills a very narrow (i.e. with diameter $\sim \operatorname{several} R_{*}$ ) hole in the disk. If the disk is optically thick, then the X-rays will be observable only when the star, or the shock front, is in the disk photosphere. Figure 1 shows the expected sequence of events, with time increasing from the top to the bottom. The star is shown as a filled circle with an arrow indicating its velocity. The optically thick denser part of the disk is shown with a darker color, and the disk photosphere with a lighter one. For convenience we call the side of the disk impacted by the star first the "front side", and the other side as the "back" one. With a yet lighter color we show the region of the disk heated by compression and by radiation to temperatures of order $\sim 10^{3}$, perhaps up to $\sim 10^{4} \mathrm{~K}$. Finally, the hottest region is the one shown with a larger circle around the star, with temperature of the order the characteristic one (Eq. (23)).

Since the star's motion through the disk is highly supersonic, the disk density remains approximately constant during the whole event except for a small region within several stellar radii from the path of the star. This means that the region of the disk pre-heated by X-rays and the shock will not have enough time to expand much and form a bulge next to the exit point of the star. The disk remains approximately plane-parallel during the star's passage through it, then. A good analogy to the situation is given by a bullet passing through a low density material (e.g. a mattress).

For the optically thick disk, the passage of the star through the disk can be divided into three stages (cf. Fig. 1):

(1) The thermal burst stage - the star is inside the optically thick part of the disk. X-rays are absorbed within the disk and re-emitted as thermal radiation in the infra-red or optical. 
This is the regime in which the star passages were extensively studied in the literature.

(2) The X-ray flare phase - the star is in the disk photosphere. The X-rays are directly visible to the observer in this phase. In the simple semi-analytical treatment of this paper, we will not make distinction between the front-side and the back-side flares, although in reality the observed emission will depend on the 3D geometry of the problem through i.e. obscuration of a part of the shock by the star itself.

(3) The "afterglow" phase - the star is many stellar radii away from the disk. The X-ray emitting gas cooled down to temperatures below $\sim 10^{7} \mathrm{~K}$ and is not observable anymore (due to high interstellar absorption to Sgr A*). A patch of the disk around the trek of the star is still "hot" compared with the unperturbed disk, and is cooling mostly by black-body emission coming out in the infra-red (e.g. Syer et al. 1991).

If the disk is optically thin this division does not apply since there is essentially only one stage.

\subsection{Division of the parameter space}

Radiation from the shocked gas is a very important facet of the given problem since it affects both the hydrodynamics and the expected spectra. Therefore, the parameter space for the problem naturally breaks down into two important cases: optically thin and optically thick (disk). We will consider these cases separately below. In addition, there is further dependence of the results on whether the cooling time of the shocked gas is shorter, comparable or longer than the adiabatic expansion time (see below). Altogether we have six cases each of which may lead to different expected flare spectra. Some of the resulting flare characteristics, such as the duration, depends mostly on geometry rather than on anything else.

\section{X-ray flares (luminosity)}

\subsection{Optically thin case}

\subsubsection{Setup}

Consider the case of a disk optically thin to X-rays as seen by the observer. The photon energy window through which Chandra and XMM are able to observe $\mathrm{Sgr} \mathrm{A}^{*}$ is the $E \sim$ $2-8 \mathrm{keV}$ range. The opacity in the disk photosphere is of course a very strong function of photon energy $E$, and at small energies, i.e. $E \sim$ few $\mathrm{keV}$, photo-absorption is very significant and the opacity is much higher than at energies $E \sim 7-8 \mathrm{keV}$. We will consider this effect in greater detail in Sect. 6.2, but for now we introduce an "effective" disk opacity, $\sigma_{\mathrm{e}}$, one which is a rough approximation to the opacity weighted with the optically thin (photon energy) spectrum of the shock. Since we expect bremsstrahlung-like hard spectra (see below), and since photo-ionization of the gas may be important (see Sect. 6.2), we estimate that $\sigma_{\mathrm{e}} \sim b \sigma_{\mathrm{T}}$, where $b$ is a factor of few, and $\sigma_{\mathrm{T}}$ is the Thomson cross section.
Within this approach, the optical depth of the disk in the relevant photon energy range along the line of sight to the observer is

$\tau \simeq \frac{b}{\cos i} \sigma_{\mathrm{T}} H n_{\mathrm{d}}$,

which yields the maximum midplane density for which the disk is still optically thin:

$n_{\mathrm{d}} \leq n_{\mathrm{ot}}=3.86 \times 10^{11} \frac{\cos i}{b} T_{2}^{-1 / 2} r_{4}^{-3 / 2}$.

Now let $\boldsymbol{v}_{*}$ be the star's 3D velocity. We define the relative velocity of the star and the gas in the disk, $v_{\mathrm{rel}}^{2}=v_{K}^{2}+v_{*}^{2}-$ $2 v_{*} v_{K} \cos \theta_{r}$, where $\theta_{r}$ is the angle between the two velocities. In general one needs to carry out calculations for arbitrary $v_{*}$ and $\theta_{r}$ and then integrate over the 3D stellar velocity distribution. Here we use the simplest approach and take the star velocity be perpendicular to the disk surface, i.e. $\theta_{r}=\pi / 2$. Since we also assume $v_{*}=v_{K}=2.1 \times 10^{8} r_{4}^{-1 / 2} \mathrm{~cm} \mathrm{~s}^{-1}$, we have $v_{\text {rel }}=\sqrt{2} v_{K}$.

We shall emphasize that in a more detailed calculation the role of the exact values for $\theta_{r}$ and $v_{*}$ will be instrumental in determining the flare characteristics. Moreover, the exact $3 \mathrm{D}$ velocity of the star will also be important. For example, if the star is moving away from the observer, then the leading edge of the bow shock is obscured from the observer by the star. This effect is not taken into account in our calculations. Thus we should keep in mind that the calculations below are meant to be representative and in reality a larger variation in the spectral and temporal characteristics of flares is expected.

\subsubsection{Flare duration and rise time}

If the disk height scale is much smaller than the radius of the star, then duration of the burst is given by $t_{\text {dur }} \simeq 2 R_{*} / v_{*}$ :

$t_{\mathrm{dur}} \sim 2 \frac{R_{*}}{v_{*}}=670 \mathrm{~s} r_{*} r_{4}^{1 / 2}$.

In the opposite case we have $t_{\mathrm{dur}} \sim H / v_{*}$

$t_{\text {dur }} \sim 2 \frac{H}{v_{*}}=5 \times 10^{4} T_{2}^{1 / 2} r_{4}^{2} \mathrm{~s}$.

Note that $H=2 R_{*}$ at $r_{4}=0.11 r_{*}^{2 / 3}$, so that for radii smaller than this one Eq. (21) should be used while for larger radii Eq. (22) is the one to use. Since the maximum luminosity reached in the bursts can be significantly higher than the quiescent Sgr A* emission, the rise time scale should be at least several times shorter than the burst duration. However it cannot be much shorter than a fraction of $R_{*} / v_{*}$, i.e. a hundred seconds for $r_{*} r_{4}^{1 / 2} \sim 1$.

\subsubsection{Characteristic temperature and time scales}

The maximum temperature to which the gas can be heated in the shock is the "characteristic" temperature defined as

$T_{\text {char }}=\frac{2}{3} \frac{\mu v_{\text {rel }}^{2}}{2 k}=1.8 \times 10^{8} r_{4}^{-1} \mathrm{~K}$, 
where we assumed that the mean particle mass in the shocked gas is $\mu=m_{\mathrm{p}} / 2$.

In a strong shock, the gas is compressed by the factor of 4 , so that the electron gas density in the shocked gas is $n_{\mathrm{sh}}=4 n_{\mathrm{d}}$. For the temperatures of interest the cooling function is dominated by the bremsstrahlung radiation. Accordingly, $\Lambda(T)=2.4 \times 10^{-27} T^{1 / 2} \mathrm{erg} \mathrm{cm}^{3} \mathrm{~s}^{-1}$ for a gas of cosmic abundance (we will include atomic emission processes in the calculation of spectra later). The cooling time of the shocked gas is

$t_{\mathrm{c}}=\frac{3 k T}{\Lambda(T) n_{\mathrm{sh}}}=5.6 \times 10^{3} \mathrm{~s}_{4}^{-1 / 2} n_{11}^{-1}$,

where we put $T=T_{\text {char }}(R)$. The radiative cooling time $t_{\mathrm{c}}$ should be compared with the adiabatic losses time scale, $t_{\mathrm{ad}}$, which is the time it takes for the shocked gas to cool off via adiabatic expansion in the disk.

$t_{\mathrm{ad}}=\frac{R_{*}}{v_{*}}=330 \mathrm{~s} r_{*} r_{4}^{1 / 2}$.

If the gas density is very high, so that $t_{\mathrm{c}} \ll t_{\mathrm{ad}}$, then the shocked gas will not be heated to the maximum temperature (Eq. (23)) because of the radiative cooling. We estimate that in this case the actual temperature of the shocked gas near the star is

$T_{\mathrm{sh}}=T_{\mathrm{char}} \frac{t_{\mathrm{c}}}{t_{\mathrm{ad}}+t_{\mathrm{c}}}$.

\subsubsection{X-ray luminosity}

The star is essentially a piston pushing the gas ahead (and aside) of it, and hence the rate at which the star makes work on the gas in the disk is approximately

$L_{w} \sim \pi R_{*}^{2} m_{\mathrm{p}} n_{\mathrm{d}} v_{\text {rel }}^{3} \simeq 7 \times 10^{34} \mathrm{erg} \mathrm{s}^{-1} n_{11} r_{*}^{2} r_{4}^{-3 / 2}$,

where $n_{11}=n_{\mathrm{d}} / 10^{11} \mathrm{~cm}^{-3}$. This is a mechanical power, and only a fraction of this energy may be emitted in X-rays. Defining $\epsilon_{\mathrm{x}}$ to be the efficiency with which mechanical power is radiated away in $\mathrm{X}$-rays with $E \gtrsim 1 \mathrm{keV}$, we write

$L_{\mathrm{xe}}=\epsilon_{\mathrm{x}} L_{w}$,

where the subscript "xe" stands for "X-ray emitted". The efficiency $\epsilon_{\mathrm{x}}$ depends on the ratio of $t_{\mathrm{c}}$ and $t_{\mathrm{ad}}$. Below we use rather rough estimates of $\epsilon_{\mathrm{x}}$; better values are unlikely to be obtained in an analytical way.

If $t_{\mathrm{c}} \ll t_{\mathrm{ad}}$, then shocked gas temperature (Eq. (26)) is smaller than $T_{\text {char }}$ due to strong radiative cooling. If $T_{\text {sh }}$ is lower than $\sim 10^{7} \mathrm{~K}$, then most of X-ray emission will be emitted at soft X-ray energies. Such emission would be absorbed in the $\sim 10^{23}$ hydrogen nuclei per $\mathrm{cm}^{2}$ column depth of the cold material in the line of sight to Sgr $\mathrm{A}^{*}$. In other words flares with $T_{\text {sh }} \lesssim 10^{7} \mathrm{~K}$ are not observable from Sgr $A^{*}$ location. However we find that the disk midplane density that explains the observed luminosity of flares in Sgr $\mathrm{A}^{*}$ best is low enough that this situation $\left(T_{\text {sh }} \lesssim 10^{7} \mathrm{~K}\right)$ is not reached. It is then appropriate to set $\epsilon_{\mathrm{x}} \simeq 1$ in the radiatively efficient case.

In the opposite limit of a very long cooling time, the shock becomes an inefficient radiator. The X-ray luminosity of the shock front around the star itself is $L \sim L_{w} t_{\mathrm{ad}} /\left(t_{\mathrm{ad}}+t_{\mathrm{c}}\right) \sim$ $L_{w} t_{\mathrm{ad}} / t_{\mathrm{c}}$. Thus in general we write

$\epsilon_{\mathrm{x}}=\frac{t_{\mathrm{ad}}}{t_{\mathrm{ad}}+t_{\mathrm{c}}}$.

In particular, in the case of $t_{\mathrm{c}} \gg t_{\mathrm{ad}}$, we have

$L_{\mathrm{xo}} \sim 4.1 \times 10^{33} \mathrm{erg} \mathrm{s}^{-1} n_{11}^{2} r_{*}^{3} r_{4}^{-1 / 2}$.

It is important to note that Eqs. (29) and (30) do not include the contribution of the shocked gas in the wake of the star. When radiative cooling is initially unimportant, the shocked gas cools first via adiabatic losses and work against the ambient unshocked gas. As the hot gas expands, the adiabatic cooling time scale $t_{\mathrm{ad}}$ eventually becomes comparable to the radiative cooling time $t_{\mathrm{c}}$, at which time most of the radiation would be emitted. If the gas temperature at that point is still larger than $10^{7} \mathrm{~K}$, then the X-ray luminosity could be as large as $L_{w}$. Unfortunately we found no reliable analytical way to accurately calculate this effect and we leave this task to future work, remembering the fact that $\epsilon_{\mathrm{x}}$ is somewhat underestimated in the $t_{\mathrm{c}} \gg t_{\mathrm{ad}}$ case.

\subsection{Optically thick disk case}

We now assume that the disk optical depth to X-rays as seen by the observer is greater than unity, and hence (cf. Eq. (20))

$n_{\mathrm{d}}>n_{\mathrm{ot}}=3.86 \times 10^{11} \frac{\cos i}{b} T_{2}^{-1 / 2} r_{4}^{-3 / 2}$.

The main difference from the optically thin case is the fact that $\mathrm{X}$-rays emitted by the shock while the star is in the disk midplane are absorbed and reprocessed into thermal radiation. As explained in Sect. 4.1, this is the regime studied intensively in the previous literature. However even in this case there will be X-ray emission when the star reaches the disk photosphere. There the density is usually low enough to preclude radiative cooling from reducing shock temperature to below $10^{7} \mathrm{~K}$. Hence the ratio of the fraction of energy emitted in X-rays to that in the optical-UV in the optically thick case is

$\frac{\int \mathrm{d} t L_{\mathrm{x}}}{\int \mathrm{d} t L_{\text {ouv }}} \leq \frac{1}{\tau_{\mathrm{d}}} \ll 1$,

where $\tau_{\mathrm{d}}$ is the optical depth of the disk to X-rays. Note also that two X-ray flares are emitted per each star-disk encounterone at the impact side and the other at the exit side of the disk (see Fig. 1), but of course the observer will see only the flare emitted from the side of the disk facing the observer.

The flare duration is now at most $\sim H / v_{*}$ because the flare from only one side is seen. In addition, the duration will be reduced by a further factor of a few if the photosphere of the disk is at several hight scales above the disk (since the disk density drops very quickly there; see Eq. (34)).

\subsubsection{X-ray light curve}

The emitted X-ray luminosity can be calculated exactly as in the optically thin case (Sect. 5.1.4) but we now take into account (i) attenuation of X-rays in the disk; and (ii) the fact that 
unshocked gas density in the disk photosphere, $n_{\mathrm{p}}$, is lower than that in the disk midplane, $n_{\mathrm{d}}$. Our treatment of the first complication is to simply introduce exponential attenuation along the line of sight. This approach underestimates the X-ray luminosity for very small $\cos i$ for which radiation scattered once in the photosphere becomes more important than the unscattered one. We defer a more careful treatment of the scattered radiation to a future paper however, to keep our calculations here as analytical and transparent as possible.

One should also keep in mind that we use the "effective" cross section for the total one (photo-absorption plus scattering), which we parameterize as $b \sigma_{\mathrm{T}}$ (see Sect. 5.1.1). Now let $\tau_{\mathrm{e}}$ be the effective total depth of the disk from the star's position $z$ to the observer. The observed X-ray luminosity is then

$L_{\mathrm{xo}} \sim L_{\mathrm{xe}} \exp \left[-\tau_{\mathrm{e}}\right]$,

where $L_{\mathrm{xe}}$ is given by Eq. (28). The gas density in the photosphere is approximately given by the Gaussian density profile:

$n(z)=n_{\mathrm{d}} \exp \left[-\left(\frac{z}{H}\right)^{2}\right]$.

The observed X-ray luminosity is now a function of time since $z=v_{* z} t$ if we define $t=0$ as the time at which the star crosses the disk midplane. Consider a front side flare - when the star is moving into the disk (i.e., see the sketch on the very bottom of Fig. 1; the observer is below the disk, at $z=-\infty$ in this case). When the star first impacts the disk photosphere, the $\mathrm{X}$-ray luminosity rapidly increases because the star moves into denser and denser environment. During this optically thin stage $L_{\mathrm{xo}} \simeq L_{\mathrm{xe}} \propto \epsilon_{\mathrm{x}} n(z)$ (Eq. (28)). Later, when the surface $\tau_{\mathrm{e}}=1$ is reached, the attenuation of X-rays by the disk matter becomes important and the luminosity dependence on the density becomes $L_{\mathrm{xo}} \propto \epsilon_{\mathrm{x}} n(z) \exp \left(-\tau_{\mathrm{e}}\right)$. Furthermore, for the Gaussian density profile

$\tau_{\mathrm{e}}(z) \simeq \frac{n(z)}{n_{\mathrm{d}}} \tau$

where $\tau$ is the optical depth to the disk midplane (Eq. (19)) and $n_{\mathrm{d}}$ is the disk midplane density. It then follows that

$L_{\mathrm{xo}}(t) \propto \epsilon_{\mathrm{x}} n(z) \exp \left[-\tau \frac{n(z)}{n_{\mathrm{d}}}\right]$.

Thus the event is indeed a flare: the luminosity first increases and then decreases quite rapidly. Note however that we implicitly assumed here that cooling time $t_{\mathrm{c}}$ is shorter than duration of X-ray flare, $t_{\text {dur }}$.

\subsection{Maximum flare $X$-ray luminosity}

The maximum observed X-ray luminosity, $L_{\max }$, for an optically thick disk, will be approximately that corresponding to $\tau_{\mathrm{e}}=1$. The gas density at which $\tau_{\mathrm{e}}=1$ is simply $n(z)=n_{\mathrm{ot}}$ as given by Eq. (20). We now substitute this value for the gas density into Eq. (28) and multiply the result by exp[-1] to get

$L_{\max }=10^{35} \epsilon_{\mathrm{x}}\left(n_{\mathrm{ot}}\right) \frac{\cos i}{b} T_{2}^{-1 / 2} r_{*}^{2} r_{4}^{-3}$ where $b \sim$ few is the ratio of the effective total cross section in the disk atmosphere to the Thomson cross section. Note that in the case of an optically thin disk the midplane density is lower than $n_{\mathrm{ot}}$ and hence the emitted X-ray luminosity is lower. Therefore Eq. (37) gives the maximum X-ray luminosity of a flare in both optically thin and thick cases.

We shall emphasize the fact that the maximum X-ray luminosity just derived is a strong function of the disk inclination angle $i$. In particular in the radiatively inefficient shock case, $\epsilon_{\mathrm{x}} \propto \cos i$ and thus $L_{\max } \propto \cos ^{2} i$. This dependence shows that if X-ray flares are indeed associated with the putative inactive disk, then the inclination angle could not be too large, i.e. the disk could not be edge-on or else the X-ray luminosity of the flares would be too small.

\section{X-ray spectrum}

\subsection{Optically thin spectrum}

In general the X-ray spectrum of a flare is complicated. The emitting region is a multi-temperature gas and only numerical calculations are likely to yield accurate spectral predictions. However single temperature optically thin spectra will provide us with certain guidance. In addition, these spectra should be of some relevance for the optically thick disk and the case of $t_{\mathrm{c}} \sim t_{\mathrm{ad}}$. Under these conditions we expect $T_{\mathrm{sh}} \sim T_{\text {char }}$ and a major fraction of the mechanical power of the shock should be "quickly" radiated in X-rays, so that the wake of the star does not make a large contribution to the emitted spectrum.

The photo-ionization parameter for the gas in the shock is $\xi \simeq L_{\mathrm{x}} / n_{\mathrm{sh}} R_{*}^{2}$ and is usually smaller than a hundred, i.e., photoionization is not important for the shocked gas, and the plasma is essentially in the coronal limit. The ionization equilibrium assumption is appropriate since the recombination time scale for the completely ionized Fe is $1 /\left(\alpha_{\mathrm{rec}} n_{\mathrm{sh}}\right) \sim 3 \mathrm{~s} n_{11}^{-1} T_{7}^{1 / 2}$. Further, the Thomson optical depth of the shocked gas is $\tau_{T} \sim$ $\sigma_{\mathrm{T}} n_{\mathrm{sh}} R_{*}=0.02 r_{*} n_{11} \ll 1$, so that the emission region is optically thin. The Compton $y$-parameter, $y \equiv 4\left(k T_{\mathrm{sh}} / m_{\mathrm{e}} c^{2}\right) \tau_{T} \ll 1$ in most realistic cases and thus Comptonization is not important for the continuum.

We computed optically thin X-ray spectra using the code XSTAR (e.g., Kallman \& Krolik 1986), assuming solar abundance of elements and that the emitting gas temperature is equal to $T_{\text {char }}(R)$. The results for three different radii, $r_{4}=0.5$, 1 and 2.5, are plotted in Fig. 2 with the dotted, thick and thin solid curves, respectively. The near infrared part of the spectra will be discussed later in Sect. 7. In Fig. 2, the top panel shows the spectra in a broad frequency range and the bottom panel concentrates on the 1-10 keV part of the spectrum.

The characteristic temperatures are $T_{\text {char }}=3.6,1.8$ and $0.73 \times 10^{8} \mathrm{~K}$ for $r_{4}=0.5,1$ and 2.5 , respectively. For such high temperatures, the X-ray spectrum is mostly bremsstrahlung emission with the great exception of the Fe $\mathrm{K} \alpha$ lines at $\simeq 6.7$ and $6.9 \mathrm{keV}$. The keV-part of the spectrum shows that the line is dominated by the He-like (i.e., the $6.7 \mathrm{keV}$ ) component for the smallest $T$ considered, whereas the $6.9 \mathrm{keV}$ component dominates for the largest value of $T$. 

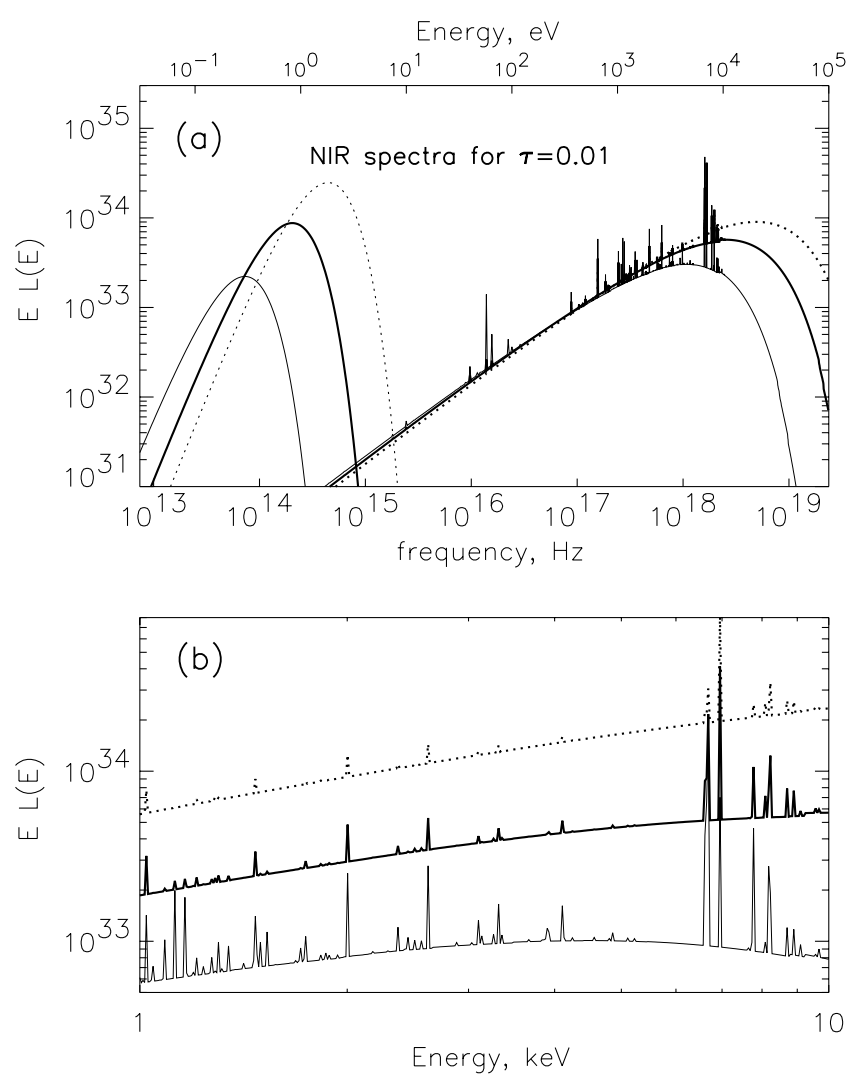

Fig. 2. Optically thin X-ray spectra for three different values of disk radius: $r_{4}=0.5,1$ and 2.5 for the dotted, solid thick and thin curves, respectively. The gas density is $n_{\mathrm{d}}=10^{11} \mathrm{~cm}^{-3}$, inclination angle $i=$ $70^{\circ}$, and the star's radius is $R_{*}=2 R_{\odot}$ for all the three cases. a) Spectra in a broad frequency range. The near infra-red curves will be explained later in Sect. 7. b) the X-ray part of the spectrum. The upper curve is shifted up by 3 whereas the lower one shifted down by the same factor for clarity. Note that, except for the brightest of the curves, the "hot" $\mathrm{Fe} \mathrm{K} \alpha$ line is clearly present.

Clearly, the integrated equivalent width $(E W)$ of $\mathrm{Fe} \mathrm{K} \alpha$ line decreases as $T_{\text {char increases ( } R \text { decreases). Another obvious }}$ trend seen in Fig. 2 is that with increase in $T_{\text {char }}$ the thermal rollover moves to higher frequencies and the spectrum begins to look more and more power-law like in the $2-8 \mathrm{keV}$ band. To quantify these trends and to compare the observed flare spectra with those predicted by our model, we defined the photon index $\Gamma$ by drawing a power-law through two line-free photon energies of $E \simeq 2.2 \mathrm{keV}$ and $7.25 \mathrm{keV}$, and also integrated over all the components of the $\mathrm{Fe} \mathrm{K} \alpha$ line to get its total $E W$. Figure 3 displays the resulting dependence of index $\Gamma$ and the $E W$ of $\mathrm{Fe} \mathrm{K} \alpha$ line on the temperature of the shocked gas.

So far only two - the brightest - of the observed X-ray flares had enough statistics to conclude that spectra during flares seem to be hard power-laws in the $2 \lesssim E \lesssim 8 \mathrm{keV}$ energy range, with the photon spectral index $\Gamma \simeq 1 \pm 0.7$ (Baganoff et al. 2001; Goldwurm et al. 2003a), and they seem to show little of an $\mathrm{Fe} \mathrm{K} \alpha$ line. Goldwurm et al. (2003b) notes that a Fe K $\alpha$ line-like feature at $\sim 6.4 \mathrm{keV}$ in their spectra is not statistically significant; but the upper limit on the $E W$ of the line is as large as $1.8 \mathrm{keV}$. From Figs. 2 and 3 it is clear that to have such hard spectra, the bright flares should have $T_{\text {sh }} \gtrsim 2 \times 10^{8} \mathrm{~K}$.

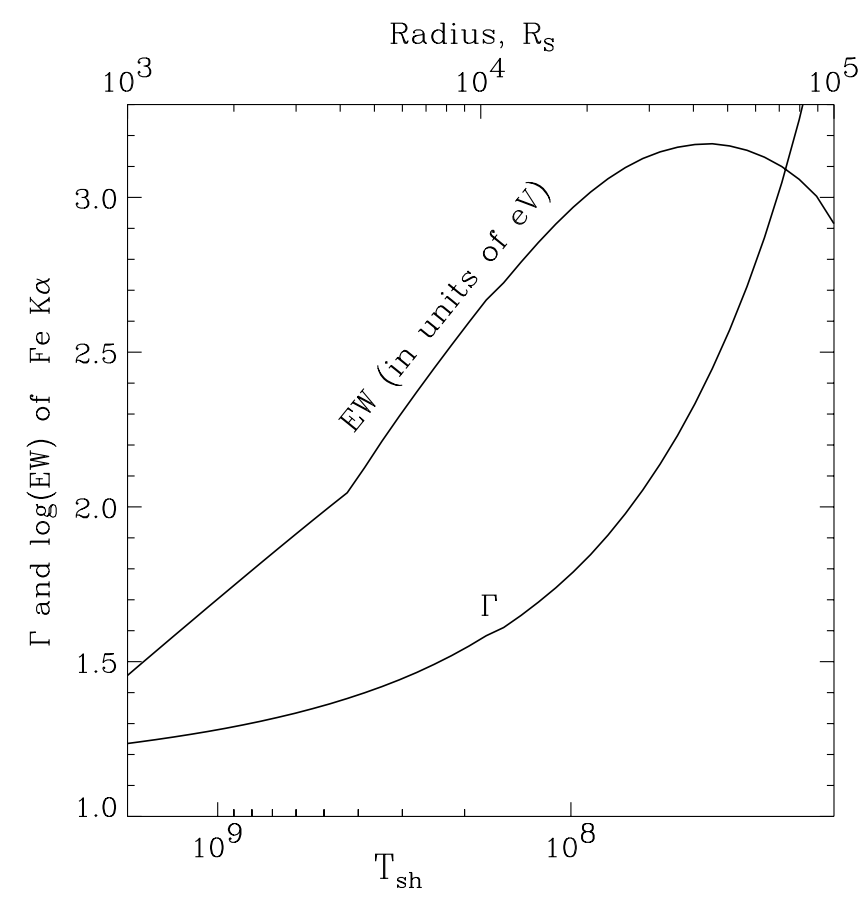

Fig. 3. a) $\log (E W)$ of $\mathrm{Fe} \mathrm{K} \alpha$ line and the $\mathrm{X}$-ray spectral index $\Gamma$ versus temperature $T_{\mathrm{sh}}$ of the emitting gas for optically thin X-ray spectra for Solar abundance of elements. If $T_{\mathrm{sh}}=T_{\mathrm{char}}(R)$, then $\Gamma$ and $E W$ may be plotted versus radius $R$, shown as the upper $x$-axis. Note that to yield Fe $\mathrm{K} \alpha E W$ and spectral index $\Gamma$ compatible with the observations of the two bright flares (Baganoff et al. 2001; Goldwurm et al. 2003a), $T_{\mathrm{sh}} \gtrsim 2 \times 10^{8} \mathrm{~K}$ or $R \lessgtr 10^{4} R_{\mathrm{S}}$.

The constraint on the $E W$ of the $\mathrm{Fe} \mathrm{K} \alpha$ line also requires similarly high values of $T_{\mathrm{sh}}$.

Finally, let us recall that in the case $t_{\mathrm{c}} \gg t_{\mathrm{ad}}$, we expect that there will be a range of temperatures that contribute to the overall spectrum, from $T=T_{\text {char }}$ in front of the star to much lower temperatures in the wake of the star. The main effect is to make the spectrum softer. Hence the minimum value of $\Gamma \simeq 1.3$ from Fig. 3 should also be the minimum value for the spectral index in the more general case.

\subsection{Absorption along the line of sight in the photosphere}

The emitted spectrum is attenuated in the atmosphere differentially for different photon energies $E$ because photon opacity is a function of $E$. The observed spectrum could be severely cut (compared to that shown in Fig. 2) at low energies $E \lessgtr 2 \mathrm{keV}$ because the opacity there is very large. Observations of at least the brightest flares show no enhancement in the column depth of the cold neutral absorber in the line of sight (Baganoff et al. 2001; Goldwurm et al. 2003a). For optically thick case, the only way to reconcile the model with the observations is if the photosphere around the star is strongly ionized and hence the absorption opacity is much smaller than that due to the cold material in the line of sight.

Baganoff et al. (2001) found that the column depth of the cold material during both quiescent and flare emission is $N_{\text {cold }} \sim 5 \times 10^{22}$ hydrogen nuclei per $\mathrm{cm}^{2}$. The column 


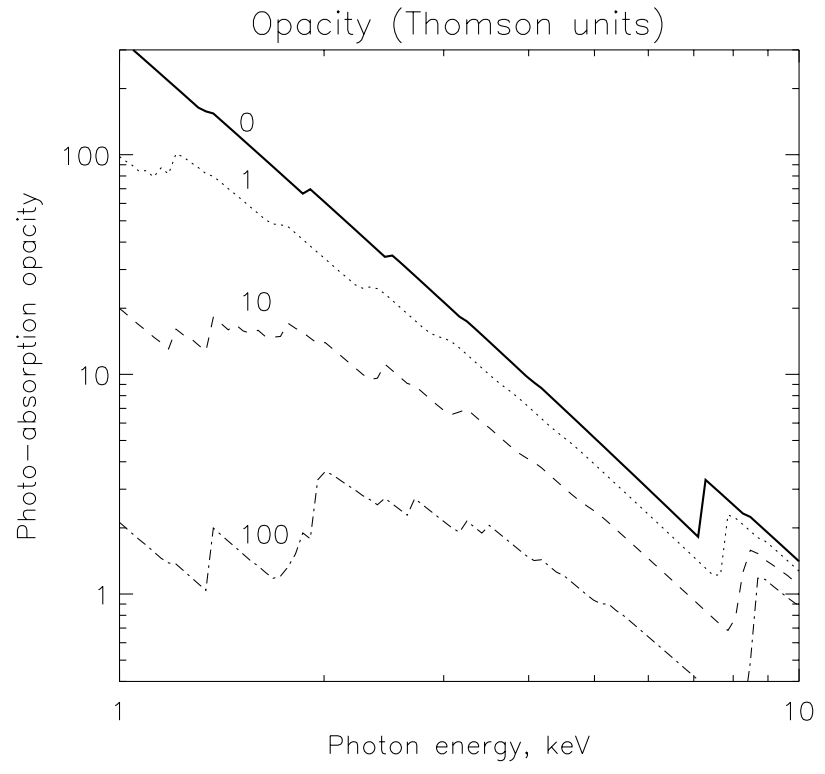

Fig. 4. Photo-absorption cross section (in units of $\sigma_{\mathrm{T}}$ ) of photo-ionized matter as a function of photon energy. Values of photo-ionization parameter $\xi$ are indicated next to the respective curves. Note a very fast decrease in the opacity with increase in the ionization parameter for $\xi \gtrsim 1$. This occurs because of photo-ionization of the elements providing the opacity.

density through which the X-rays pass during the maximum X-ray observed emission is around $N_{\text {ion }} \sim 1 / b \sigma_{\mathrm{t}} \sim 5 \times 10^{23}$. We then need the absorption opacity of the ionized photosphere to satisfy

$\frac{\sigma_{\text {ion }}(E)}{\sigma_{\text {cold }}(E)} \ll \frac{N_{\text {cold }}}{N_{\text {ion }}} \sim 0.1$,

where $\sigma_{\text {cold }}$ is the absorption cross section of the cold gas which is much larger than $\sigma_{\mathrm{T}}$, the Thomson cross section. For energy $E$ in Eq. (38) it is sensible to take $E \sim 2 \mathrm{keV}$ because the cold gas opacity is largest at the smallest energies in the $\mathrm{keV}$ band, and so the measurement of $N_{\text {cold }}$ is really dominated by the lowest energy bin (see Fig. 3 in Baganoff et al. 2001).

The ionization state of the disk atmosphere illuminated by $\mathrm{X}$-rays from the shock is controlled by photo-ionization rather than by collisional ionization since the gas temperature is low, i.e. $T \sim T_{\text {ir }} \sim 10^{3}-10^{4}$ Kelvin. Using XSTAR once again, we calculated the ionization state of plasma kept at $T=10^{4} \mathrm{~K}$ and illuminated by X-rays with spectrum similar to those shown in Fig. 2. The resulting absorption cross section as a function of photon energy is plotted in Fig. 4 for different values of photoionization parameter which is defined as $\xi \equiv 4 \pi F_{\mathrm{x}} / n_{0}$ where $F_{\mathrm{x}}$ is the integrated X-ray flux. The solid curve labelled " 0 " is calculated for a very small $\xi$ and yields the "cold" absorption cross section $\sigma_{\text {cold }}(E)$. The figure shows that the condition specified by Eq. (38) is fulfilled when ionization parameter exceeds about 10 .

We estimate the average ionization parameter in the photosphere around the star as

$\xi \sim \frac{L_{\mathrm{xe}}}{n_{0}(H / 2)^{2}}$, where $L_{\mathrm{xe}}$ is the emitted X-ray luminosity at the maximum light, which is given by $L_{\max } \mathrm{e}^{1}$ (see Sect. 5), and as representative distance from the star $H / 2$ is chosen. For example, the case of an optically thick disk with adiabatic losses dominating the radiative ones gives

$\xi \sim 0.042 \frac{\cos i}{\sigma_{\mathrm{e}}} T_{2}^{-3 / 2} r_{*}^{3} r_{4}^{-5}$.

Clearly strong enough ionization of $\xi \gtrsim 10$ requires the two bright detected X-ray flares to happen at small values of $r_{4}$ and/or be produced by large stars with $r_{*} \gg 1$. Taking a concrete example of a 5 Solar radii star, we find that $\xi$ exceeds 10 for radii $r_{4} \lesssim 0.6$. Note that the photo-ionization parameter $\xi$ at the time of maximum light in X-rays is a function of the disk inclination angle, so once again we see the dependence of the results on the disk orientation to us (cf. Sects. 5.2.1 and 5.1.1).

We shall emphasize that the constraint $\xi \gtrsim 10$ is important only if the disk is somewhat optically thick. The disk may be optically thin to X-rays which we find quite likely for the innermost disk region where the bright flares should be taking place.

\section{Flares in the near infrared}

\subsection{Optically thick case}

First consider the simplest case of a disk optically thick in the near infrared. X-rays emitted by the shock while inside the optically thick disk are easily absorbed by the cold un-shocked gas and reprocessed into thermal radiation. Therefore we assume that all the work done by the star on the cold gas is emitted as a blackbody radiation in this case and that the luminosity is equal to $\simeq L_{w}$ (Eq. (27)). The radiation runs ahead of the shock wave, heating and ionizing the gas in the disk. The region affected by the radiation is roughly the cube with size $\sim 2 H$. The temperature of the region can be found by assuming that the cooling time of the disk region warmed up by the X-rays from the shock, $t_{\mathrm{cd}}$, is shorter than $H / v_{*}$. This implies that $L_{w} \sim \sigma_{B} T_{\text {ir }}^{4} \times 2(2 H)^{2}$ and

$T_{\text {ir }}=1.78 \times 10^{3} \mathrm{~K}\left[\frac{n_{11} r_{*}^{2}}{T_{2}}\right]^{1 / 4} r_{4}^{-9 / 8}$

(index ir stands for "infra-red"). If $t_{\mathrm{cd}}>t_{\mathrm{dur}}$, then the thermal luminosity is lower than $L_{w}$.

As remarked in Sect. 4, the pre-heated gas cannot expand much during the star's passage through the disk. The observed flare luminosity in near infra-red is then reduced by the disk projection effect: $L_{\mathrm{obs}}=L_{w} \cos i$. The near infra-red $K$-band seems to yield the tightest constraints on the theoretical models (see, e.g., Hornstein et al. 2002). The corresponding reference wavelength is $2.2 \mu \mathrm{m}$ and the frequency is $v_{0}=1.36 \times 10^{14} \mathrm{~Hz}$. The predicted observed luminosity is then $v L_{v}=4 \pi \times 4 H^{2} \cos i v B_{v}\left(T_{\mathrm{ir}}\right)$ :

$v L_{v}=7.8 \times 10^{36} \cos i T_{2} r_{4}^{3} \frac{\bar{v}^{4}}{e^{x}-1}$,

where $\bar{v} \equiv v / v_{0}$ and $x \equiv h v / k T_{\text {ir }}$, and where we assumed that the distance to the Galactic Center is $D=8.0 \mathrm{kpc}$. Note that the 
factor of $4 \pi$ above arises due to the usual assumption that the emitter (the disk) emits isotropically in the full $4 \pi$ steradians. Also note that the dependence on the disk density is "hidden" in the temperature $T_{\text {ir }}$ of the emission and that $x=\bar{v}\left(h v_{0} / k T_{\text {ir }}\right)=$ $\bar{v}\left(6.5 \times 10^{3} \mathrm{~K} / T_{\text {ir }}\right)$.

The luminosity given by Eq. (42) is quite large. The current best limit on the quiescent emission of $\mathrm{Sgr} \mathrm{A}^{*}$ at $2.2 \mu \mathrm{m}$ is about $2 \mathrm{mJy}$ (dereddened; Hornstein et al. 2002). In terms of $v L_{v}$, the quiescent limit of $2 \mathrm{mJy}$ corresponds to about $2 \times 10^{34} \mathrm{erg} \mathrm{s}^{-1}$. Hornstein et al. (2002) note that the limits on random 3-hour flares is about a factor of 10 higher, i.e. $\simeq 2 \times 10^{35} \mathrm{erg} \mathrm{s}^{-1}$. Hence an optically thick (in NIR) disk in Sgr A* would produce NIR flares strong enough to be detected by now.

\subsection{Optically thin disk}

Previously we pointed out that no optically thick disk can exist in Sgr A* already based on its quiescent spectrum - or the disk would reprocess too large a fraction of the visible and UV stellar flux into the infrared band and would violate the observed upper limits (see Sect. 2.2). These constraints limit the infrared optical depth of the disk to $\tau_{\text {ir }} \lesssim 0.01$. If the opacity of the disk grains were grey, the grain temperature would equal to the effective one (that is $T_{\mathrm{ir}}$ ). In this approximation the luminosity of the flare in the NIR is simply reduced by the factor $\tau_{\text {ir }}$ :

$v L_{v}=7.8 \times 10^{34} \frac{\tau_{\text {ir }}}{0.01} \cos i T_{2} r_{4}^{3} \frac{\bar{v}^{4}}{e^{x}-1}$.

Figure 2, panel a, shows examples of such spectra for a star with radius $R=2 R_{\odot}$. Note that the NIR flares are weak enough to be missed. Also note that the value of the dust optical depth, assumed to be equal to 0.01 for all frequencies, is likely to be smaller than that of the disk in quiescence. The stellar passage through the disk may in fact destroy the dust (see below) in which case the NIR luminosity will be reduced even further.

It is tempting at this point to make a connection between $\Sigma$, the disk surface density given by Eq. (11), and the optical depth in the NIR using the standard interstellar dust opacity. For example, at $2.2 \mu \mathrm{m}$, the dust extinction is about $5 \times 10^{-22} \mathrm{~cm}^{2}$ per hydrogen atom (see Fig. 2 in Voshchinnikov 2003). Thus with $n_{\mathrm{d}} \sim 10^{11} \mathrm{~cm}^{-3}$ the disk would be optically thick at $2.2 \mu \mathrm{m}$ for radii greater than about $10^{3} R_{\mathrm{S}}$. However the dust opacity in the disk is likely to be reduced compared with the interstellar values by the grain growth. Following Spitzer (1978), we estimate that the grain radius $a$ increases at the rate $\mathrm{d} a / \mathrm{d} t \simeq 10^{-3} \zeta_{a} n_{11}^{-1} \mathrm{~cm} /$ year, where $\zeta_{a} \lesssim 1$ is the average fraction of atoms that stick to the grain in the course of collisions with the grain. This rate is very large so there may be no small grains in the disk. On the other hand dust grains will be destroyed or broken up into smaller grains by dust-dust collisions.

Dust grains are also destroyed during star passages. The gas temperature in the cylinder with radius $H$ around the star's trek in the disk is given by Eq. (41). This temperature is high enough to destroy all or most of the dust species. Therefore we may define a "dust destruction" time scale, $t_{\mathrm{dd}}$, as time needed to cover all of the disk area within radius $R$ by star-disk collisions
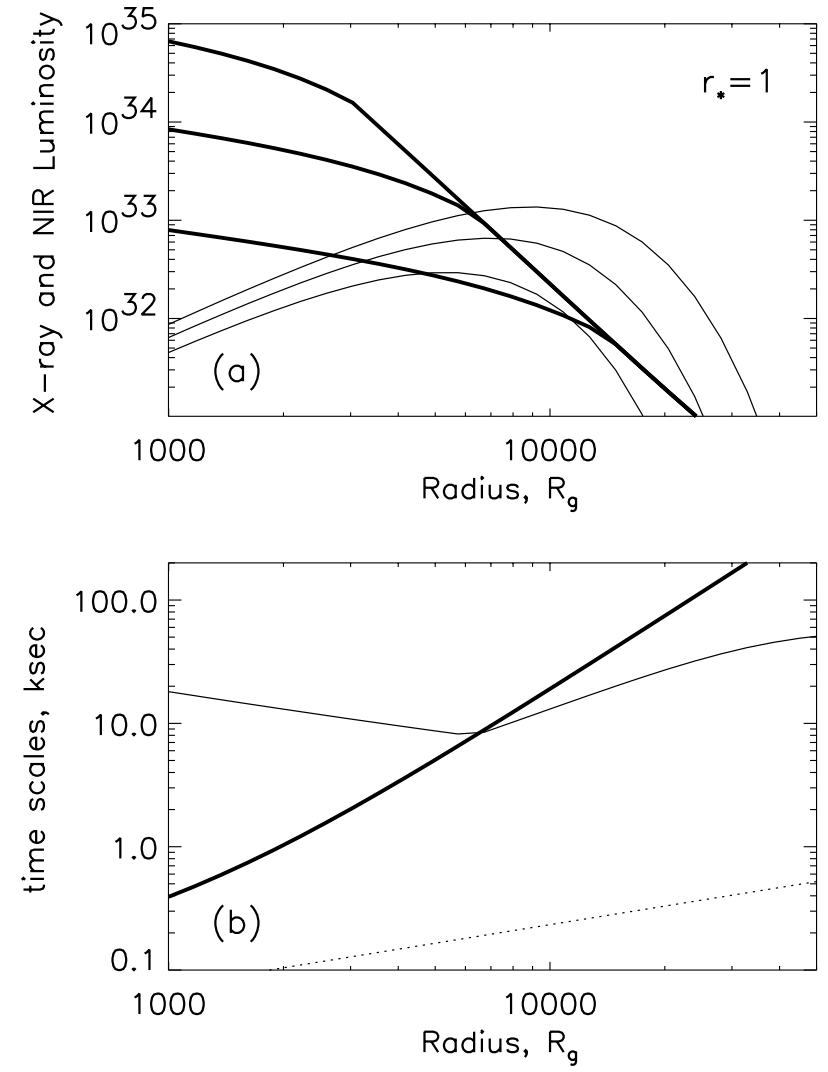

Fig. 5. Flare properties for $R_{*}=R_{\odot}$, disk inclination angle of $i=75^{\circ}$ and $b=2$ (see Eq. (37)). a) Maximum X-ray (thick) and NIR (thin) luminosity during flares as a function of radius for three different values of disk midplane densities, $n_{\mathrm{d}}=3 \times 10^{10}, 10^{11}$ and $3 \times 10^{11}$ hydrogen nuclei per $\mathrm{cm}^{3}$. For NIR luminosities, we assume that the dust optical depth is 0.01 , as in Eq. (43). b) Burst duration (thick solid), adiabatic expansion time (dotted) and the cooling time (thin solid). Only the latter depends on the disk density, which was chosen to be $n_{\mathrm{d}}=10^{11}$ for the thin solid curve.

(each collision "covers" area $\sim \pi H^{2}$ of the disk). Using the rate of the star-disk passages calculated in Sect. 3.1, we obtain

$t_{\mathrm{dd}}=\frac{\pi R^{2}}{\dot{N}(R) \pi H^{2}} \simeq 2 \times 10^{4}$ years $T_{2}^{-1} r_{4}^{-1}$.

This time is very short compared with the viscous time scales for the cold disk $\left(\sim 10^{7}-10^{8}\right.$ years $)$.

\section{Typical "weak" and typical "strong" flares}

We now consider two important cases. The first one is the passage of a "low"-mass star with $R_{*}=R_{\odot}$ through the disk for a range of midplane disk densities $n_{\mathrm{d}}$, and for different radii $R$. The second case is that of a "large" star, with $R=5 R_{\odot}$, passing through the same disk.

The low-mass star case is analyzed in Fig. 5. The midplane disk density $n_{\mathrm{d}}$ is chosen to be $n_{\mathrm{d}}=3 \times 10^{10}, 10^{11}$ and $3 \times 10^{11}$ hydrogen nuclei per $\mathrm{cm}^{3}$. The $\mathrm{X}$-ray luminosities are shown with thick curves while the near infrared $2.2 \mu$ luminosities are plotted with thin curves in panel a. Larger values of these luminosities correspond to larger values of the midplane density. Panel $b$ of the figure shows three time scales - the 

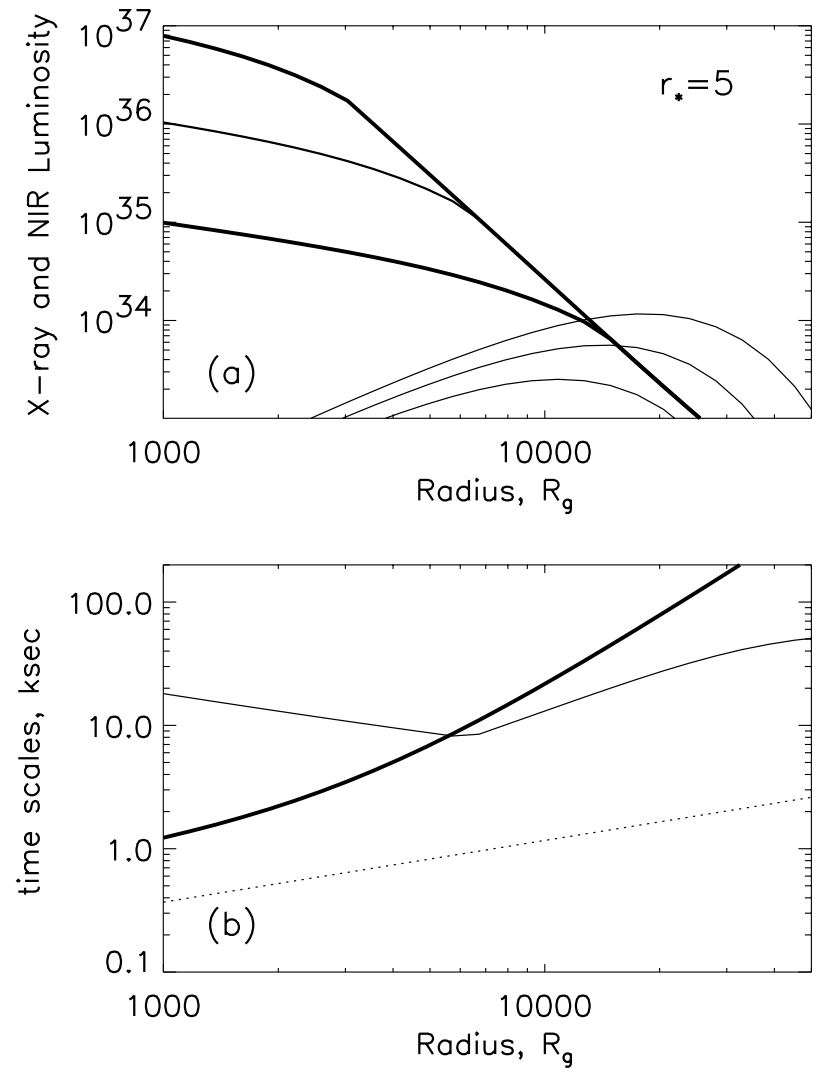

Fig. 6. Same as Fig. 5 but for a high mass star with $R=5 R_{\odot}$. Note the change in the luminosity scale; X-ray flares are roughly 100 times brighter than they were in Fig. 5. The NIR emission increased by a much smaller factor mostly because it is in the Rayleigh-Jeans regime.

duration (Eq. (22)), cooling (Eq. (24)) and adiabatic expansion time (Eq. (25)). Out of these only the cooling time depends on the disk density, which was chosen to be $n_{\mathrm{d}}=10^{11} \mathrm{~cm}^{-3}$ for the respective curve in panel $b$.

The X-ray luminosity curves have a common asymptote at large radii, which is simply the optically thick case given by Eq. (37). It is very important to note that in this optically thick limit the X-ray luminosity is an extremely strong function of radius: $L_{\mathrm{xo}} \propto R^{-3}$. Given the fact that quiescent $\mathrm{X}$-ray luminosity of $\mathrm{Sgr} \mathrm{A}^{*}$ is about $L_{\mathrm{q}} \sim 10^{33} \mathrm{erg} \mathrm{s}^{-1}$, it is clear that flares from solar-sized stars will not be observable for $R \gtrsim 10^{4} R_{\mathrm{S}}$. A comparison with the case of a large star, Fig. 6, shows that flares from these are observable up to radii of $\sim$ few $\times 10^{4} R_{\mathrm{S}}$, i.e. just a factor of few higher. This conclusion is independent of the disk midplane density: the flare X-ray luminosity at a given radius $R$ cannot exceed $L_{\max }$ (Eq. (37)). Thus we conclude that as far as X-ray flares are concerned, only the "inner" disk region with $R<$ few $\times 10^{4} R_{\mathrm{S}}$ matters. At small disk radii, the disk becomes optically thin to X-rays along the line of sight to the observer. At this point the X-ray luminosity curves (solid thick curves) change their slope.

"Large" stars produce higher luminosities than do "small" ones, in both optically thin and thick regimes. This is so because the total work done by the star on the disk is proportional to the area of the star (Eq. (27)), plus radiative cooling is actually more efficient for larger stars (Eq. (30)). There are of course fewer high mass stars than there are low mass ones in the Sgr A* star cluster (Genzel et al. 2003), and hence high luminosity flares should be a minority.

The duration of typical flares is in the range of one to tens of kilo seconds. Flares with longer durations are not detectable because they are too weak; flares shorter than about a thousand seconds require a small $R_{*}$ (see Eq. (21)) and thus may be too week as well. In addition, the cooling time at small $R$ becomes longer than $t_{\text {dur }}$ (Fig. 5b).

The near infrared ( $2.2 \mu$ here) emission is quite weak in all the models, at or below the $\sim 2 \times 10^{35} \mathrm{erg} \mathrm{s}^{-1}$ upper limit set by Hornstein et al. (2002). This is partially because the black-body emission is a rather narrow feature and in most cases the $2.2 \mu$ is either on the Wien exponential tail or on the Rayleigh-Jeans part of the curve. We should also note that the NIR luminosities are calculated under the optically thick assumption which may be violated for the smallest values of the disk surface density $\Sigma$. However most likely optically thin spectra would peak in the optical band again producing little NIR flux to be detected (see Sect. 7).

The cyclotron frequency in the hot blob is about $1 \mathrm{GHz}$, assuming equipartition magnetic field. However, its intensity is many orders of magnitude larger than the Rayleigh-Jeans intensity at this frequency, so this emission is completely selfabsorbed and therefore impossible to observe. It is unlikely that non-thermal mechanisms could significantly alter this conclusion. Therefore our model predicts no radio-flares correlated with X-ray flares (but star-disk interactions may have indirect implications for the jet and hence some radio variability may in fact be due to stars; see also Nayakshin \& Sunyaev 2003).

\section{Comparison with observations of X-ray flares}

Observational facts for X-ray flares from Sgr A* may be summarized as following (Baganoff et al. 2001, 2003b; Goldwurm et al. 2003a). The maximum X-ray luminosity detected so far is $L_{\mathrm{x}} \sim 10^{35} \mathrm{erg} \mathrm{s}^{-1}$. Such strong flares are relatively rare; i.e. there is probably $\sim 5-10$ times more of the weaker $L_{\mathrm{x}} \lesssim 10^{34} \mathrm{erg} \mathrm{s}^{-1}$ flares. Counting all detectable flares, there is about one flare per day on average. There seems to be no NIR or radio counterparts to the flares (Baganoff et al. 2003b; Hornstein et al. 2002). In particular the radio source has never varied by more than a factor of 2 during many years of observations (Zhao et al. 2001). The NIR limits on random $\sim 3$ hour flares are about $\sim 2 \times 10^{35} \mathrm{erg} \mathrm{s}^{-1}$, much too tight for many existing models of Sgr A* flares (Hornstein et al. 2002). The spectra appear to be harder than those during quiescence and show little if any $\mathrm{Fe} \mathrm{K} \alpha$ line emission (both Baganoff et al. 2001 and Goldwurm et al. 2003a,b X-ray spectra contain weak emission-line like features at energies not too far from the expected $\mathrm{Fe} \mathrm{K} \alpha$ line but detailed modeling is difficult due to poor statistic. Goldwurm et al. 2003b put an upper limit of $1.8 \mathrm{keV}$ on a $6.4 \mathrm{keV} \mathrm{Fe} \mathrm{K} \alpha$ line feature in their spectrum of an X-ray flare). In addition Porquet et al. (2003) reported a very bright X-ray flare observed with XMM that showed a soft X-ray spectrum and an excess of soft X-ray absorption. 
The following flare characteristics appear to have a reasonable explanation in our model (we place the items in order from model independent to less so):

- (i) Frequency of flares, i.e. number of flares per day, which in our model is around few per day (see Sect. 3.1 and in particular Eq. (16)).

- (ii) Duration of X-ray flares is in the range of $\sim$ one to tens of kilo-seconds.

- (iii) X-ray luminosities of observed flares in the peak of their light-curves are consistent with the maximum allowed luminosities in our model (Eq. (37) with $\epsilon_{\mathrm{x}}$ given by Eq. (29)). Namely, the observed luminosities are best explained by an optically thin disk with midplane density of order $10^{11}$ hydrogen nuclei per $\mathrm{cm}^{3}$.

- (iv) X-ray flares from similar star-disk encounters in the nuclei of other LLAGN. On the one hand, the maximum X-ray luminosity of flares at a given dimensionless distance $r_{4}$ from the black hole, and a given disk surface density $\Sigma$, scales as (see Eq. (33)): $L_{\mathrm{x}} \propto n_{\mathrm{ot}}^{2} \propto H^{-2} \propto 1 / M_{\mathrm{BH}}^{2}$. That is, more massive black holes produce weaker $\mathrm{X}$-ray flares. Further, the background quiescent X-ray luminosity of confirmed LLAGN is always larger than that of Sgr A*, usually by some 3-6 orders of magnitude. On the other hand, some nearby Galaxies, e.g. M 31, are not detected in X-rays, whereas M 32 has just been detected with $L_{\mathrm{X}} \sim 10^{36} \mathrm{erg} \mathrm{s}^{-1}$ (Ho et al. 2003). We believe that due to a wide distribution in flare properties, there are flares brighter than that, although they are infrequent. Thus such bright flares could be detectable in the nearby galaxies, especially with future XEUS and Constellation-X missions.

- (v) X-ray spectra are expected to have a distribution of properties and it is actually difficult to define a "typical" flare (see Sect. 10). Therefore observations of flares should be compared with theoretical predictions in a statistical sense rather than on the basis of a single observation.

- (vi) If the disk is moderately optically thick in relevant frequency (e.g. soft X-rays), then spectra of flares may show an additional absorption compared with that in the interstellar medium between Sgr A* and the observer.

- (vii) Near infrared (NIR) luminosity of flares produced by the shock around the star is around or smaller than the current detection limit at $2.2 \mu$ (see Sect. 7). However a more detailed modeling of the star-disk passages is needed to cover the range of theoretical possibilities.

- (viii) No flare radio emission is expected in our model during the star-disk impact. Such emission is completely selfabsorbed in our model.

\section{Shortcomings and a broader picture}

Theoretical spectra from star-disk flares depend on a number of factors that will vary from flare to flare. In this paper we presented a rather simplified treatment of the problem. However, for a comparison with observations, it is important to be aware of these important complications since this shows the resulting diversity of flare properties.
Geometry. The exact geometry of the star-disk passage is crucial for quantitatively accurate results. Namely, the angle that the star makes with the disk rotation velocity, $\theta_{\mathrm{r}}$, controls the total mechanical energy deposition rate, the duration of the $\mathrm{X}$-ray burst, and the relative position of the bow shock with respect to the star as seen by observer. (Here we simply assumed $\theta_{\mathrm{r}}=\pi / 2$.) For example, if the disk is optically thick, then appearance of flares with oppositely directed stellar velocities, e.g., $\boldsymbol{v}_{*}$ and $-\boldsymbol{v}_{*}$, is completely different.

Stellar orbits. We assumed that the stars are on circular Keplerian orbits. In fact many of the observed stars have highly elliptical orbits, many with $e \simeq 0.9$ (e.g. Genzel et al. 2003; Ghez et al. 2003b). Therefore one needs to also allow for variation in the absolute value of $v_{*}$.

Massive stars. Although outnumbered by the less massive ones, the high mass stars will produce the brightest flares. The effects of very bright stars on the disk before and during the star-disk passage may change the flare characteristic substantially. In particular, the stellar radiation may be reprocessed into the NIR band giving rise to NIR flares. A related complication is the fact that bright stars produce powerful winds. The latter may be as important in shocking and heating the disk as the stellar surface itself for high wind loss rates.

Stellar coronae and photospheres. In this paper we assumed that the star is a rigid solid body. However, stellar coronae and the photospheres may be important for the gas dynamics during the star-disk passages. If luminosity of the radiation emitted by the shock is much larger than the intrinsic stellar luminosity, then the star's photosphere will be strongly heated. Therefore a part of the photosphere may be blown away during the disk passage, releasing extra energy and changing the emitted spectrum. This may in fact give rise to NIR flares that are stronger than those discussed in the paper.

Unusual abundance of chemical elements. The abundance of the putative disk in Sgr $\mathrm{A}^{*}$ need not be Solar. In fact if the disk is currently fed by the hot helium rich stars, then the hydrogen in the disk may be under-abundant. In addition, the disk may contain a much higher mass fraction of dust than the normal $\sim 0.01$ fraction due to evaporation of gas from the disk by different forms of external heating.

Compact objects. Additionally, Sgr A* star cluster may host a number of compact objects - white dwarfs, neutron stars and stellar mass black holes. These would produce flares quite different from those that we have studied here. Especially one has to worry about the presence of $\sim 10 M_{\odot}$ black holes since these are massive and are expected to sink into the central parts of the $\mathrm{Sgr} \mathrm{A}^{*}$ star cluster. The radiation emitted by a stellar mass black hole passing through the disk would be powered mostly by accretion of gas on this black hole rather than shocks, and hence produce different spectra. 


\section{Discussion}

In this paper we proposed that the observed X-ray flares in Sgr A* are emitted by bow shocks around stars when the latter pass through an inactive disk. Such a disk has recently been invoked by Nayakshin (2003) to explain the quiescent spectrum of Sgr A*. The disk may be a remnant of the past accretion and star formation activity in our Galactic Center (Sect. 2.1; see also Levin \& Beloborodov 2003). Due to quiescent Sgr A* spectral constraints, the disk temperature is restricted to be of order $10^{2} \mathrm{~K}$, whereas the size of the disk is at least $10^{4}$ Schwarzschild radii. The disk should be optically thin in mid infrared frequencies or else it would violate the existing limits on the quiescent Sgr A* luminosity (see Sect. 2.2; Cuadra et al. 2003). From modeling X-ray flare luminosities we concluded that the disk midplane density is of order $10^{11}$ hydrogen nuclei per $\mathrm{cm}^{3}$. Such a disk is quite "light" - it is not a subject to gravitational instability and it will not gravitationally influence the dynamics of Sgr A* stellar cluster significantly (see Sect. 2).

Using the latest results on star content in Sgr A* stellar cluster (Genzel et al. 2003), we calculated the rate of the star-disk crossings to be few per day (Sect. 3). We then found that the rate of star-disk crossings in the inner disk region with size $R_{\mathrm{ii}} \sim 10^{3} R_{\mathrm{S}}$ is so large that the angular momentum transfer there will be dominated these crossings rather than disk viscosity. Therefore the disk innermost region may be hotter than the inactive cool disk at larger radii.

In the optically thin disk case, X-ray emission during the star-disk passage is directly observable. In the optically thick case, on the other hand, X-rays from the shock front are reprocessed in the infrared frequencies while the star is inside the disk. X-ray flares are emitted only when the shock arrives at the disk photosphere. We found that there exists a maximum $\mathrm{X}$-ray luminosity that can be emitted for given stellar parameters (Eq. (37)). This then shows that flares occurring at radii greater than $\sim$ few $\times 10^{4} R_{\mathrm{S}}$ are too weak to be detectable on the background X-ray emission of Sgr $\mathrm{A}^{*}$. This fact may explain why the flares have durations in the range from one to tens of kiloseconds (see Sect. 8).

The predicted spectra range from very hard free-free spectra with spectral index $\Gamma \sim 1.5$ to softer ones if the emitting gas is multi-temperature (Sect. 6.1). The hardest spectra are also predicted to show little $\mathrm{Fe} \mathrm{K} \alpha$ line emission whereas softer flares should generally show the helium and hydrogen like components of such lines. Finally, these lines could be significantly red- or blue-shifted depending on whether the flare occurs on the approaching or the the receding side of the disk: $\Delta E_{\text {line }} \sim\left(v_{K} / c\right) \sin i 6.7 \mathrm{keV}=50 \sin i r_{4}^{-1 / 2} \mathrm{eV}$. The model also predicts an extra soft absorption in X-ray if the disk has large enough column depth. Note that such atomic features in Sgr A* spectra are unlikely to be produced in the context of the three other existing models for Sgr A* flares (Markoff et al. 2001; Liu \& Melia 2002; Narayan 2002, Sect. 5.3). In all of these models flares come from a region very close to the black hole, i.e., $\sim 10 R_{\mathrm{S}}$, where all the important chemical species are completely ionized because the gas is very hot. We also found that similar X-ray flares resulting from star-disk passages in other LLAGN and nearby inactive Galaxies are rather hard to detect except for the most nearby and low black hole mass sources.

Note added in proof: Half way through the refereeing process we have learned from Prof. R. Genzel about an exciting discovery of a set of NIR flares from Sgr A* that in many respects remind the X-ray flares. In terms of our models, these flares are best explained as the emission of the transiently heated stellar photo-spheres during the disk passages. The observed NIR periodicity may be a signature of hydrodynamical oscillations excited in the upper layers of the stars by the collision with the disk.

Acknowledgements. We are very grateful to R. Genzel, R. Schödel, T. Ott, P. Predehl and D. Porquet for the opportunity to learn their latest unpublished results, as well as many stimulating discussions. We also appreciate comments of T. Alexander, A. Goldwurm \& A. Ghez. Finally, comments from many of our colleagues at MPA in Garching, and especially C. P. Dullemond, have also been very useful. M. Morris, the referee, is thanked for a very detailed and useful referee report on this long paper.

\section{References}

Alexander, T. 1999, ApJ, 527, 835

Armitage, P. J., Zurek, W. H., \& Davies, M. B. 1996, ApJ, 470, 237

Bahcall, J. N., \& Wolf, R. A. 1976, ApJ, 209, 214

Baganoff, F. K., Bautz, M. W., Brandt, W. N., et al. 2001, Nature, 413, 45

Baganoff, F. K., Maeda, Y., Morris, M., et al. 2003a, ApJ, 591, 891

Baganoff, F. K., et al. 2003b, Astron. Nachr., 324, S1 (2003), Special Supplement, The central 300 parsecs of the Milky Way, ed. A. Cotera, H. Falcke, T. R. Geballe, S. Markoff

Balick, B., \& Brown, R. L. 1974, ApJ, 194, 265

Blandford, R. D., \& Begelman, M. C. 1999, MNRAS, 303, L1

Bobinger, A., Horne, K., Mantel, K.-H., \& Wolf, S. 1997, A\&A, 327, 1023

Bower, G. C., Wright, Melvyn, C. H., Falcke, H., \& Backer, D. C. 2003, ApJ, 588, 331

Cannizzo, J. K. 1998, in Wild Stars in The Old West, ed. S. Howel, E. Kuulkers, \& C. Woodward (San Francisco: ASP), ASP Conf. Ser., 137, 308

Coker, R., Melia, F., \& Falcke, H. 1999, ApJ, 523, 642

Coker, R., \& Melia, F. 2000, ApJ, 534, 723

Cuadra, J., Nayakshin, S., \& Sunyaev, R. 2003, A\&A, 411, 405

Falcke, H., \& Melia, F. 1997, ApJ, 479, 740

Falcke, H., \& Markoff, S. 2000, A\&A, 362, 113

Frank, J., \& Rees, M. J. 1976, MNRAS, 176, 633

Gammie, C. F. 2001, ApJ, 553, 174

Genzel, R. 2000, in Proc. of the Star2000 Meeting (Heidelberg, March 2000), ed. R. Spurzem [astro-ph/0008119]

Genzel, R., Schödel, R., Ott, T., et al. 2003, ApJ, 594, 812

Ghez, A. M., Becklin, E., Duchêne, G., et al. 2003a, Astron. Nachr., 324, S1 (2003), Special Supplement, The central 300 parsecs of the Milky Way, ed. A. Cotera, H. Falcke, T. R. Geballe, S. Markoff [astro-ph/0303151]

Ghez, A. M., Duchêne, G., Matthews, K., et al 2003b, ApJ, 586, L127

Goldreich, P., \& Lynden-Bell, D. 1965, MNRAS, 130, 97 
Goldwurm, A., Brion, E., Goldoni, P., et al. 2003a, ApJ, 584, 751

Goldwurm, A., Brion, E., Goldoni, P., et al. 2003b, Astron. Nachr., 324, S1 (2003), Special Supplement, The central 300 parsecs of the Milky Way, ed. A. Cotera, H. Falcke, T. R. Geballe, S. Markoff [astro-ph/0303583]

Hagio, F. 1987, PASP, 39, 887

Ho, L. C. 1999, ApJ, 516, 672

Ho, L. C. 2003, to appear in Active Galactic Nuclei: from Central Engine to Host Galaxy, ed. S. Collin, F. Combes, \& I. Shlosman (San Francisco: ASP) [astro-ph/0303059]

Ho, L. C., Terashima, Y., \& Ulvestad, J. S. 2003, ApJ, 589, 783

Hornstein, S. D., Ghez, A. M., Tanner, A., et al. 2002, ApJ, 577, L9

Ivanov, P. B., Igumenshchev, I. V., \& Novikov, I. D. 1998, ApJ, 507, 131

Karas, V., \& Subr, L. 2001, A\&A, 376, 686

Kallman, T. R., \& Krolik, J. H. 1986, NASA/GSFC Laboratory for High Energy Astrophysics special report

Kolykhalov, P. I., \& Sunyaev, R. A. 1980, SvALett., 6, 357

Levin, Y., \& Beloborodov, A. M. 2003, ApJ, 590, L33

Liu, S., \& Melia, F. 2002, ApJ, 566, L77

Loewenstein, M., Mushotzky, R. F., Angelini, L., et al. 2001, ApJ, $555, \mathrm{~L} 21$

Markoff, S., Falcke, H., Yuan, F., \& Biermann, P. L. 2001, A\&A, 379, L13

Melia, F. 1994, ApJ, 426, 577

Melia, F., Falcke, H. 2001, ARA\&A, 39, 309

Miyoshi, M., Moran, J., Herrnstein, J., et al. 1995, Nature, 373, 127

Narayan, R., \& Yi, I. 1994, ApJ, 428, L13

Narayan, R., Yi, I., \& Mahadevan, R. 1995, Nature, 374, 623

Narayan, R. 2002, Lighthouses of the Universe, ed. M. Gilfanov, R. Sunyaev, \& E. Churazov (Springer)

Nayakshin, S., \& Sunyaev 2003, MNRAS, 343, L15
Nayakshin, S. 2003, in Astron. Nachr., 324, S1 (2003), Special Supplement, The central 300 parsecs of the Milky Way, ed. A. Cotera, H. Falcke, T. R. Geballe, \& S. Markoff [astro-ph/0302420]

Norman, C., \& Silk, J. 1983, ApJ, 266, 502

Ostriker, J. P. 1983, ApJ, 273, 99

Perry, J. J., \& Williams, R. 1993, MNRAS, 260, 437

Porquet, D., Predehl, P., Aschenbach, B., et al. 2003, A\&A, 407, L17

Proga, D., \& Begelman, M. C. 2003, ApJ, 592, 767

Ptak, A., Serlemitsos, P., Yaqoob, T., \& Mushotzky, R. 1999, ApJS, 120,179

Quataert, E., Di Matteo, T., Narayan, R., \& Ho, L. 1999, ApJ, 525, L89

Quataert, E. 2003, in Astron. Nachr., 324, S1 (2003), Special Supplement, The central 300 parsecs of the Milky Way, ed. A. Cotera, H. Falcke, T. R. Geballe, \& S. Markoff

Rauch, K. P. 1995, MNRAS, 275, 628

Reid, M. J., Readhead, A. C. S., Vermeulen, R. C., \& Treuhaft, R. N. 1999, ApJ, 524, 816

Shakura, N. I., \& Sunyaev, R. A. 1973, A\&A, 24, 337

Schödel, R., Ott, T., Genzel, R., et al. 2002, Nature, 419, 694

Shlosman, I., \& Begelman, M. C. 1989, ApJ, 341, 685

Syer, D., Clarke, C. J., \& Rees, M. J. 1991, MNRAS, 250, 505

Vilkoviskij, E. Y. 1983, Astron. Lett., 9, 405

Vilkoviskij, E. Y., \& Czerny, B. 2002, A\&A, 387, 804

Voshchinnikov, N. V. 2002, in Optics of Cosmic Dust, ed. G. Videen \& M. Kocifaj (Kluwer), 1-36 [astro-ph/0303162]

Yuan, F., Markoff, S., \& Falcke, H. 2002, A\&A, 383, 854

Zentsova, A. S. 1983, Ap\&SS, 95, 11

Zhao, J., Bower, G. C., \& Goss, W. M. 2001, ApJ, 547, L29

Zurek, W. H., Siemiginowska, A., \& Colgate, S. A. 1994, ApJ, 434, 46 